\title{
UNIVERSITY OF KWAZULU NATAL
}

A robust air refractometer for accurate compensation of the refractive index of air in everyday use

Oelof Abraham Kruger

2016 


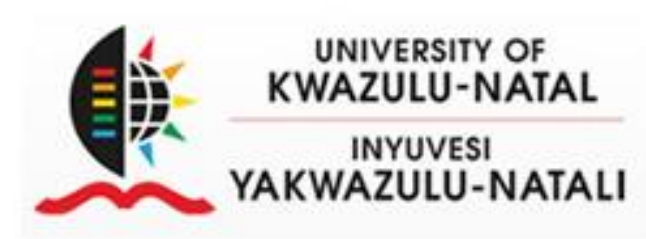

\section{A robust air refractometer for accurate compensation of the refractive index of air in everyday use}

\section{Oelof Abraham Kruger}

215081719

December 2016 


\section{A robust air refractometer for accurate compensation of the refractive index of air in everyday use}

\section{Oelof Abraham Kruger \\ 215081719 \\ December 2016}

Thesis presented in partial fulfilment of the requirements for the degree of Master of Science in Physics

School of Chemistry and Physics, Discipline of Physics

College of Agriculture, Engineering and Science

University of KwaZulu-Natal (PMB), Private Bag X01, Scottsville, 3209, South Africa

Supervisor:

Professor Naven Chetty 


\section{Declaration}

This thesis describes the work undertaken at the University of KwaZulu-Natal under the supervision of Prof N. Chetty between March 2015 and December 2016.

I declare the work reported herein to be own research, unless specifically indicated to the contrary in the text.

Signed:

Student: O. A. Kruger

On this day of .2016

I hereby certify that this statement is correct.

Signed:

Supervisor: Prof N. Chetty

On this day of .2016 


\section{ACKOWLEDGMENTS}

A heartfelt thank you to my family for coping with all the time I spent away from home for enduring my temperaments when I was home and working on the project.

I wish also to thank my supervisor, Naven Chetty who has played a vital role in not only supervising the project, but also my overall guidance and support on all aspects of the projects. Further thanks to him for the original motivation to commence with the project in the first place and to further my studies. My utmost admiration is extended.

Lastly, but certainly not least, I express my gratitude to NMISA, for providing the environment and funding to undertake this project and further my studies. 


\section{Abstract}

While the definition of the metre is based on the speed of light in a VACUUM, most dimensional measurements are performed using a laser interferometer in AIR. Velocity of light (V.O.L) compensation needs to be applied to the velocity of the laser light for accurate measurements of the speed of light to be approximated in vacuum.

Best practice is generally through the use of a weather station method where the ambient conditions are measured i.e., air temperature, air pressure, humidity and carbon dioxide levels. Then, using a modified Edlen's equation, corrections for the wavelength of the laser in air compared to the vacuum wavelength is made. This method is however only accurate to $3 * 10^{-8}$ and thus other techniques are required to improve the accuracy of the measured results.

With this in mind, this work focusses on investigations into the V.O.L compensations both to improve on the accuracy of the Edlen equation method in everyday use, and to verify the accuracy of the current weather station systems method utilised.

A refractometer that allows for V.O.L. compensation measurements was developed, tested and verified. The system was designed to be simple and cost-effective for use in everyday dimensional measurements but with high accuracy. Achieved results show that although simple in design, the refractometer is accurate to approximately $1 * 10^{-8}$ which meets our initial condition for design.

This indicates that using a refractometer design that is low cost, allows measurements to make V.O.L. compensations more accurate than the weather station method. Further this method compares favourably with other refractometer systems designed to perform similar functions although with much higher cost and complexity of use. 


\section{Table of Contents}

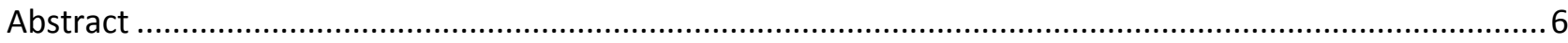

Chapter 1: Impact of Dimensional metrology and the requirement for accurate measurements .................10

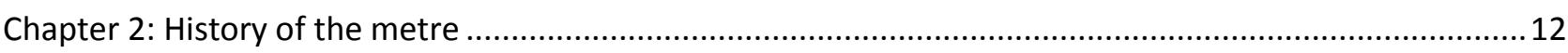

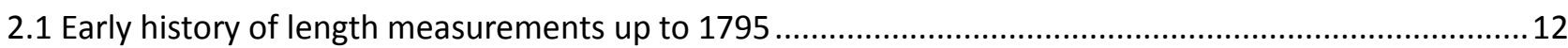

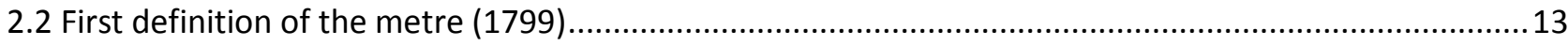

2.3 The International Bureau of Weights and Measures (BIPM) ............................................................

2.4 The wavelength definition, a great step forward (1927) ...................................................................... 14

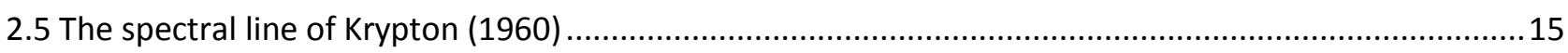

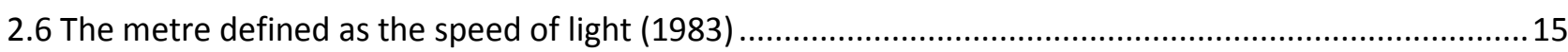

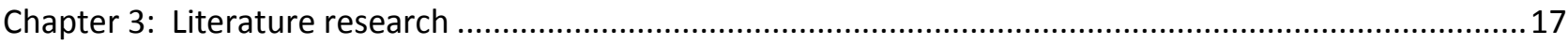

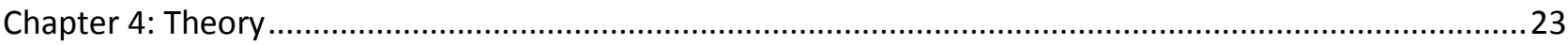

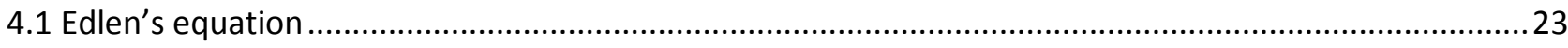

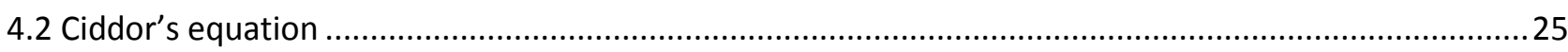

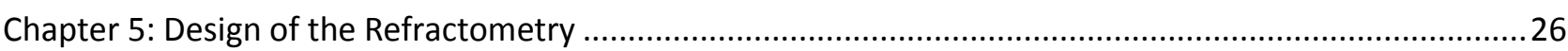

5.1 Principles of length measurements using distance laser interferometers ............................................26

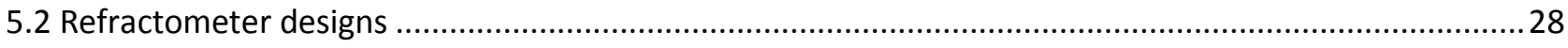

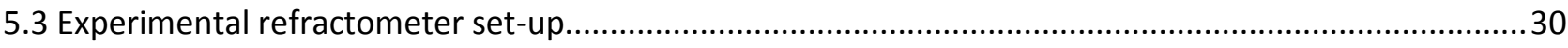

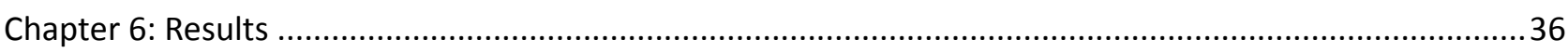

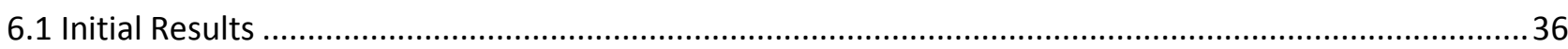

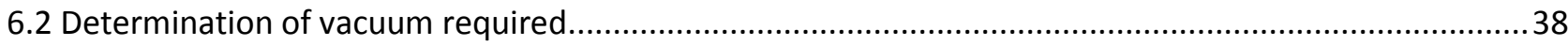

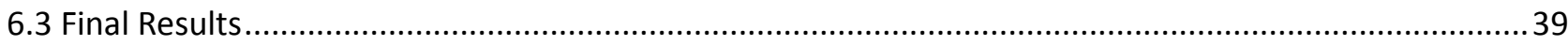

6.4 Investigation of temperature, pressure and humidity fluctuations .................................................... 40

6.5 Comparison of refractometer results to commercial systems............................................................. 42

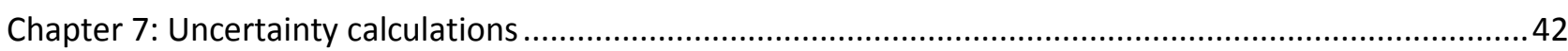

7.1 Refractive index uncertainty of the Edlen weather station method.......................................................42

7.2 Refractive index uncertainty due to length of vacuum tube .................................................................43

7.3 Refractive index uncertainty due to laser reading ............................................................................. 43

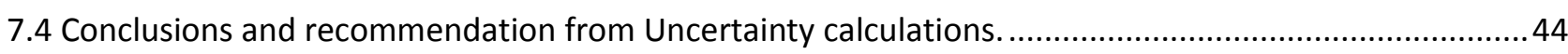

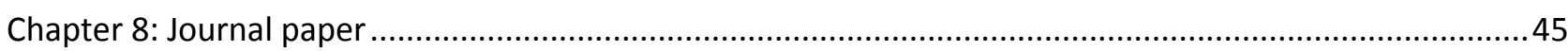

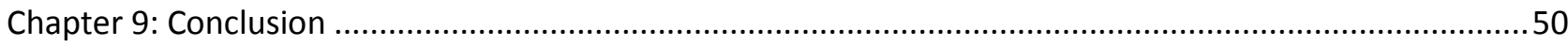

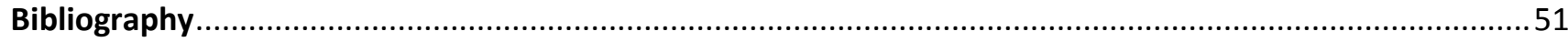

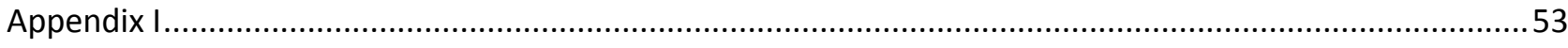

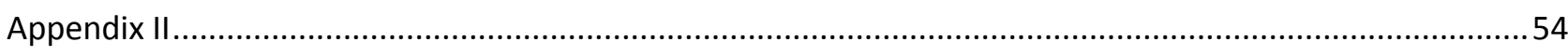




\section{TABLE OF ABBREVIATION}

BIPM - Bureau International des Poids et Mesures

$\mathrm{CCL}$ - Consultative Committee for Length

CGPM - General Conference on Weights and Measures

HP - Hewlett Packard company, now known as Agilent

ISO - International Organization for Standardization

GUM - Guide to the Expression of Uncertainty in Measurement

NMIs- National Metrology Institutes

ppm - parts per million

SI - International System of Units

V.O.L. - Velocity Of Light 


\section{LIST OF FIGURES, TABLES AND GRAPHS}

Figure 1: Determining the average foot of a wood engraving from the year 1576.

- Figure 2: Measuring the length of the meridian passing through Dunkirk and Barcelona during the years 1792 to 1798 .

$>$ Figure 3: The metre, originally defined by a length marked on a bar such as shown in the background, is currently defined via the speed of light and realized via the wavelength and frequency of lasers, as illustrated by the laser beam in the foreground.

Figure 4: Typical Output spectrum of a Krypton lamp

$>$ Figure 5: The main iodine features observed with a $633 \mathrm{~nm}$ HeNe laser. The components are from the 11-5 R(127) transition. The separation between components $d$ and $g$ is approximately $40 \mathrm{MHz}$.

> Figure 6: Patented Hewlett- Packard designed wavelength tracker.

$>$ Figure 7: Schematic of a dual interferometer with one interferometer measuring sample displacements while the other measures refractive index fluctuations.

$>$ Figure 8: Operation of a heterodyne laser system

$>$ Figure 9: Heterodyne laser system, beat frequency versus velocity

> Figure 10: Patented refractometer showing the vacuum etalon which is moved through the laser beams.

$>$ Figure 11: Schematic diagram of proposed Refractometer.

$>$ Figure 13: Schematic diagram of layout of the vacuum tube showing the layout of the vacuum pump and the gauge.

$>$ Figure 14: Vacuum tube with two optical flats bonded to the sides

$>$ Figure 15: Picture of completed refractometer

$>$ Figure 16: Vacuum tube with layout changed to move the vacuum tube to the other inlet of the vacuum tube.

Table 1: Results for the refractometer data compared to the modified Edlen equation using weather station measurement calculations.

$>$ Table 2: Results of the last reading taken by the refractometer compared to different calculation methods.

Graph 1: The laser reading measured relative to different levels of vacuum.

$>$ Graph 2: The refractive index plotted relative to the air temperature arranged from low to high.

$>$ Graph 3: The refractive index plotted relative to the air pressure arranged from low to high.

$>$ Graph 4: The refractive index plotted relative to the air relative humidity arranged from low to high. 


\section{Chapter 1: Impact of Dimensional metrology and the requirement for accurate measurements}

Metrology is the scientific study of measurement and in Lord Kelvin's words: "I often say that when you can measure what you are speaking about, and express it in numbers, you know something about it; but when you cannot measure it, when you cannot express it in numbers, your knowledge is of a meagre and unsatisfactory kind; it may be the beginning of knowledge, but you have scarcely in your thoughts advanced to the state of Science, whatever the matter may be." (1)

Metrology is divided into three categories with different levels of complexity and accuracy $(2 ; 3)$ :

i) Scientific metrology deals with the organisation and development of measurement standards and with their maintenance (highest level).

ii) Industrial metrology has to ensure the adequate functioning of measurement instruments used in industry, in production and testing processes, for ensuring quality of life for citizens and for academic research.

iii) Legal metrology is concerned with measurements where these influence the transparency of economic transactions, particularly where there is a requirement for legal verification of the measuring instrument.

The need for accurate length and dimensional measurements and a clear definition for the metre are needed to support these three categories.

Dimensional metrology supports these areas:

i) Research into better length measurements pushing for higher accuracy in dimensional measurements are hindered by problems of fundamental metrology. These include establishing accurate standards of derived units (pressure, flow, capacitance, irradiance, etc.) or measuring fundamental physical constants such as Planck's constant, Boltzmann's constant and the universal gravitational constant. Mass measurements using a watt balance is used to measure the Planck constant or, in the new $\mathrm{SI}$, to realise the mass unit. Capacitance standards (calculable capacitor, or the silicon sphere are used for determination of the Avogadro constant. All these measurements standards benefit from optical interferometry at the sub-nanometer level to measure linear dimensions or displacements more accurately (4).

ii) Metrology in production engineering must be fast, robust and automated. Optical methods have been around for a long time but the rapid progress in the development of optical components and increased computing power makes the development using optical measurements more attractive. Schwenke (5) provides the detail of areas in which it is progressing and hence supporting the manufacturing process using optical engineering. Estler (6) provides a summary of the impact and progress of metrology with special focus on large scale metrology.

iii) Length measurements are required in legal metrology where everything that is sold per metre requires accurate measurements to fix the correct price. Just imagine if a kilometre of cable of an expensive material for example optical fibre or a $50 \mathrm{~mm}$ 
diameter steel wire rope is measured wrong by a few percent, the cost to the actual measurement could be dramatically skewed.

This need is all the more important for South Africa in the global economy, since without it, parts manufactured in South Africa would not fit together with parts manufactured anywhere else in the world. This becomes more important as McKinsey (7) points out that manufacturing is the largest potential income generator with over R500bn market size.

All of these measurements are performed using optical techniques and require a standardised international scale of length. When optical techniques are employed the accurate compensation to the refractive index of air is critical. This will be explained in greater detail in Chapter 2 and 3 .

Apart from the impact of the refractive index measurements affecting all length measurements, it is also used in measurements of the refractive index of optics and to determine the sugar content in wine (8). Another impact requiring the accurate measurement of refractive index of air has been in the calculation of density of air required for buoyancy corrections in mass calibration. Picard $(9 ; 10)$ describes a comparison to determine the density of moist air using three different methods. The most widely used method for determining the density of air is where $p$ the pressure, $t$ the thermodynamic temperature, $x_{v}$ the mole fraction of water vapour, $M_{a}$ the molar mass of dry air, $M_{v}$ the molar mass of water, $Z$ the compressibility factor and $R$ the molar gas constant, are measured and used to calculate the air density.

Yet another method described in the literature is the use of a refractometer specially designed for applications similar to that discussed in this work. The changes in air density are determined with high precision using an optical method based on the correlation between air density and refractive index. The air buoyancy artefacts method has also been discussed in some detail in the literature $(8,9,11)$. This method is based on the weighting of two artefacts having the same nominal mass and the same surface area but two different volumes. Two weightings are used, the one in air and the other in a vacuum. The difference divided by the volume calculates the density of air. The work was to demonstrate the validity of using the air buoyancy artefacts method and not to determine the most suitable method for determining the density of air. 


\section{Chapter 2: History of the metre}

\subsection{Early history of length measurements up to 1795}

The ancients developed moderately accurate ways to measure four quantities; Length, Area, Volume, and Weight or Mass, which they did not distinguish between. They used time to measure large lengths and areas, for example, the journey was so many hours, days or moons. An acre was the amount of land that a team of oxen would plough in a day, and the length of the furrow was called the furlong $(11 ; 12)$.

Many ancient units of measurement were derived from body parts or easily obtainable materials. We still speak of the foot and the hands when measuring length. Some more units based on other body parts are less obvious. The inch was based on the last joint of the thumb. The yard was the distance from the tip of the nose to the end of the fingers with the right arm out-stretched. The digit was one finger width. The palm was four digits. The hand was five digits. The cubit, is the length of the arm from the elbow to the tip of the middle finger $(11 ; 12)$.

Needless to say, these measurements can vary widely from person to person. An example of allowing for this variance can be seen in the 12th century Scottish definition for the inch, which was taken to be the thumb width from the average of three men - one big, one medium and one small. Attempts to standardise the units of length date back many hundreds of years, for example, in the 16th century. A definition of the average foot was used for trading purposes. Surveyors would wait outside a church on Sunday, and line up the first 16 men that came out to measure their average feet as in Figure $1(11 ; 12)$.

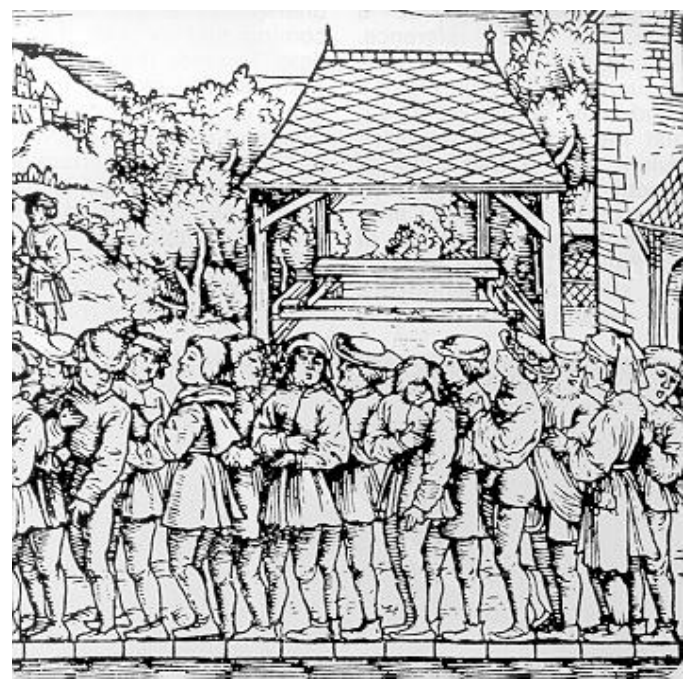

Figure 1: Determining the average foot, a wood engraving from the year 1576. 


\subsection{First definition of the metre (1799)}

It had long ago been realised that a universal standard of measurement for length was needed. In 1791 the French National Assembly decided in favour of a standard that would be one ten millionth of a meridian quadrant known as the metre. The survey which established the length standard was made from Dunkirk, in France, to Barcelona, in Spain (Figure 2). The survey was long, fairly difficult to do accurately and was carried out during a time when France and Spain were heading to war. Once the result of the measurements was finalised, the provisional standard, made in 1793, was replaced by the true definitive metre standard, a gauge block made of pure platinum, in the form of a bar having a rectangular cross-section, whose size from end to end standardised the metre. In 1799 the metre standard was placed, together with the kilogram standard, also made from platinum, in the National Archives Paris.

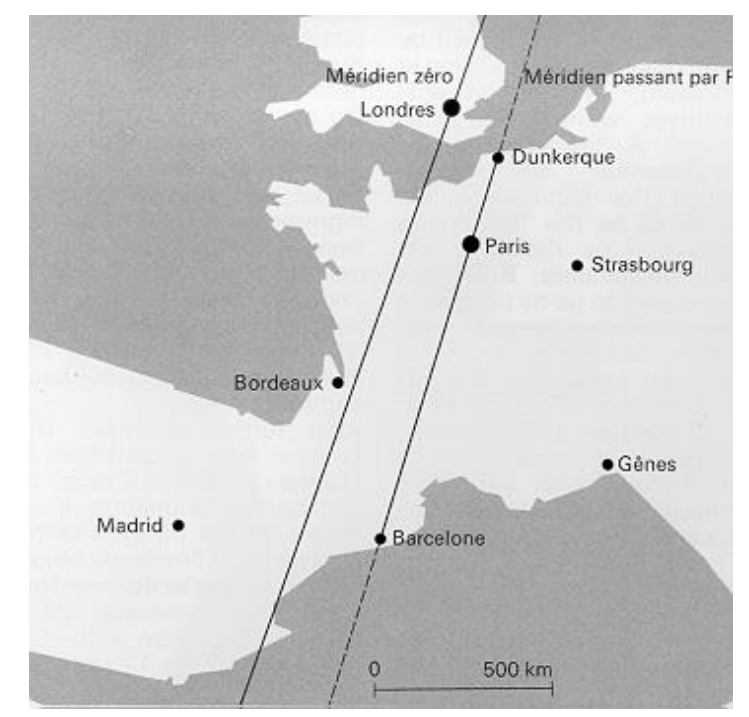

Figure 2: Measuring the length of the meridian passing through Dunkirk and Barcelona during the years 1792 to 1798 .

\subsection{The International Bureau of Weights and Measures (BIPM)}

However these bars were difficult to reproduce and in 1889 a number of new platinum-iridium metre bars were produced and one of these, No 6, replaced the International Prototype Metre and became the new standard $(11 ; 12 ; 13)$. The new international prototype metre is quite different from its predecessor. Apart from the metal composition, the most important difference is that it was used as a line standard instead of an end standard. That is instead of end to end the distance between two lines engraved on it. The bar is of X-shaped cross-section $20 * 20 \mathrm{~mm}$ overall, giving maximum rigidity with minimum mass (Figure 3). The first session of the General Conference in October 1889 confirmed the prototype definition of the metre and granted it legal validity. The uncertainty value for the prototype metre was high at approximating $0,2 \mu \mathrm{m}$. 


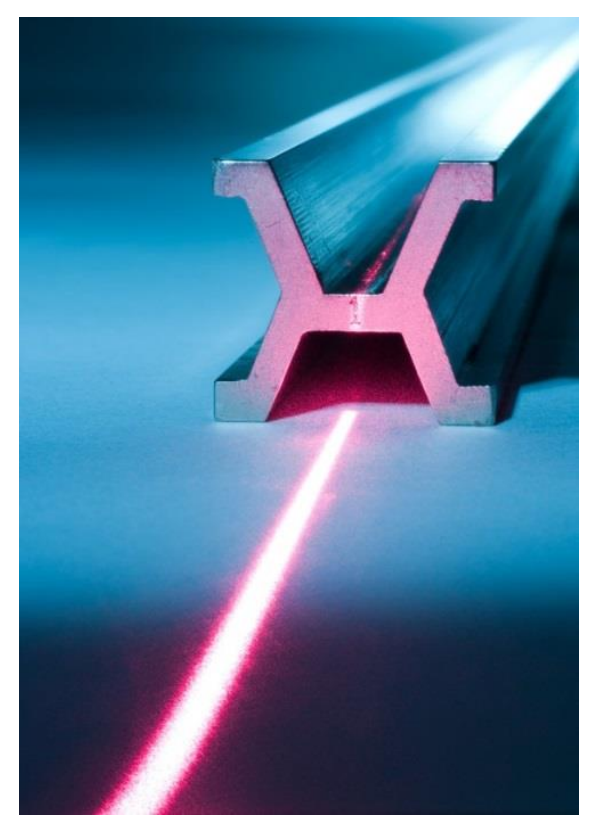

Figure 3: The metre, originally defined by a length marked on a bar such as shown in the background.

\subsection{The wavelength definition, a great step forward (1927)}

The question then arose what would occur if the prototype metre was damaged or even destroyed in the event of fire, war or natural catastrophe? The logical sequence was to adopt a standard length based on some natural law phenomenon, which could be referred to and reproduced at any time and place on the earth $(11 ; 12)$. It was of course also essential that the new definition of the metre should represent a clear advanced obtainable accuracy, relative to the line standard which was then in use.

It was in 1889, the very year that the international prototype metre was legally sanctioned, that work began on finding a replacement. At the 7th General Conference on Weights and Measures (CGPM) in 1927, the metre was defined as 1552 164,13 times the wavelength of the red spectral line of cadmium in the atmosphere at a pressure of $1013 \mathrm{mbar}$ and a temperature of $15^{\circ} \mathrm{C}$. It was from this definition that wavelength was used to define the metre and hence the accurate measurement of the refractive index of air became critical $(11 ; 12)$. 


\subsection{The spectral line of Krypton (1960)}

The technical procedures for spectral measurements were not universally accessible at the time, therefore it was decided to continue using the international prototype as the primary reference for as long as it should be necessary (14). This ambiguity was to continue for more than 30 years. It was not until 14th October 1960 that the 11th General Conference on Weights and Measures finally agreed that the definition of the metre represented by the 85 year old line standard was insufficiently accurate. The new definition, which comprised of the orange spectral line of Krypton, was the following: "the metre is the length equal to 1650763,73 wavelengths in vacuum of the radiation corresponding to the transition between the levels $2 p_{10}-5 d_{5}$ of the Krypton- 86 atom" (14).

This new definition of the metre allows lengths to be defined with a measurement uncertainty of only $4 \mathrm{~nm}$ per metre $\left(4 * 10^{-9}\right)$.

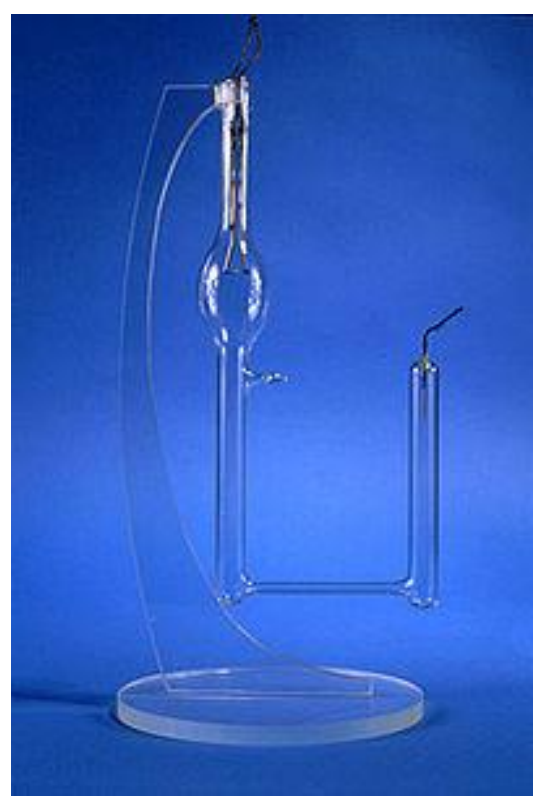

Figure 4: Typical Krypton lamp as used to define the metre between 1960 and 1983 (14).

\subsection{The metre defined as the speed of light (1983)}

In 1960, the world was presented with a new type of optical technology that was referred to as "the solution in search of a problem" $(11 ; 12)$. This device was the laser; an acronym for Light Amplification by Stimulation Emission of Radiation. The previous definition for the metre lasted only 23 years. On 20th October 1983, at the 17th General Conference on Weights and Measures the new definition for the metre as "the length of path travelled by light in vacuum during a time interval of (1/299 792458$)$ of a second" was adopted.

In essence there is no longer a physical standard for length. This strange definition actually defines the metre more accurately, because the time interval can be measured with an accuracy of better 
than $1 * 10^{-13}$. Wilke (15) explains once the physicists could measure the speed of light more accurately than they could measure the metre itself, the days of the metre was numbered.

The 1889 definition of the metre, based on the international prototype of platinum-iridium, was replaced by the 11th General Conference on Weights and Measures (CGPM) in 1960, using a definition based on the wavelength of krypton 86 radiation. This change was adopted in order to improve the accuracy to which the definition of the metre could be realised, the realisation being achieved using an interferometer with a travelling microscope to measure the optical path difference as the fringes when counted. In turn, this was replaced in 1983 by the 17th CGPM. The metre is since 1983 defined as "the length of path travelled by light in vacuum during a time interval of $1 / 299792458$ of a second $(16 ; 17 ; 18)$.

This allows each country to realise the metre though a reproduction of the SI definition of the metre. lodine-stabilised HeNe lasers are mainly used in many national metrology laboratories in each country and measurement institutes for the realisation of the SI unit of length, the metre. Such systems offer an accurate and effective way for traceability of length and dimensional measurements.

One of the recommended radiations is the $633 \mathrm{~nm}$ light from an iodine-stabilised HeNe laser, to which is currently assigned an overall uncertainty of $10 \mathrm{kHz}$ which relates to approximating 2 parts in $10^{11}(17)$. This applies to the radiation of a HeNe laser with an internal iodine cell, stabilized using the third harmonic detection technique, subject to the conditions:

- cell-wall temperature $(25 \pm 5)^{\circ} \mathrm{C} 3$;

- cold-finger temperature $(15.0 \pm 0.2){ }^{\circ} \mathrm{C}$;

- frequency modulation width, peak-to-peak, $(6.0 \pm 0.3) \mathrm{MHz}$;

- one-way intracavity beam power (i.e. the output power divided by the transmittance of the output mirror) $(10 \pm 5) \mathrm{mW}$ for an absolute value of the power shift coefficient $1.0 \mathrm{kHz} \mathrm{mW}-1$.

The absorption lines, as shown in Figure 5 are from the 11-5 $R(127)$ transition. The separation between components $\mathrm{d}$ and $\mathrm{g}$ is approximately $40 \mathrm{MHz}$. The advantage of these is that all the lines can in turn be used to measure against a commercial laser and the repeatability calculated. These lodine-stabilised HeNe lasers are in-turn used to calibrate commercial lasers system such as the systems used later in this research.

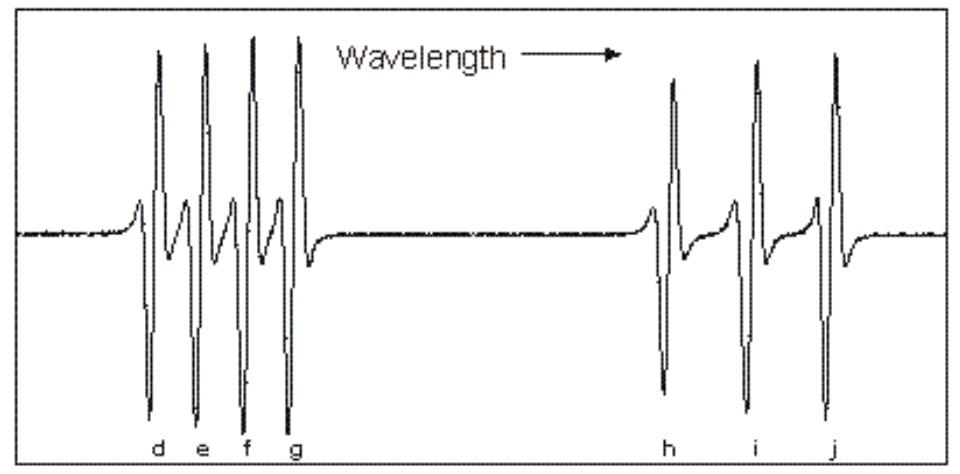

Figure 5: The main iodine features observed with a $633 \mathrm{~nm}$ HeNe laser. 


\section{Chapter 3: Literature research}

As described in the previous Chapter, the metre, is since 1983, defined as "the length of path travelled by light in vacuum during a time interval of $1 / 299792458$ of a second $(16 ; 18 ; 17)$.

The need for accurate length measurements and subsequently a definition is needed throughout the modern world as captured in the strategy of the Consultative Committee for Length (4). Much of industry and technology relies on these measurements as described in Chapter 2. With dimensional metrology's ever increasing accuracy, it necessitates relooking at the uncertainty contributors to dimensional measurements.

The previous Chapter describes the history of the metre and how we came to the metre defined as the speed of light in a vacuum. However most of the dimensional and length measurements are performed in air. The refractive index in air differs with different environmental conditions (air pressure, air temperature, humidity and $\mathrm{CO}_{2}$ ) and the refractive index of air at specific conditions have to be calculated to make corrections to the speed of light in air, compared to the speed of light in a vacuum (19).

To make this correction the refractive index of air at the specific environmental conditions must be calculated. To calculate the refractive index, the speed (velocity) of light in a vacuum $c$, is divided with $v^{\prime}$, the velocity of light in air at the time the measurements are taken.

There are two methods to determine the refractive index. In the first method the environmental conditions namely, air temperature, air pressure, relative humidity and $\mathrm{CO}_{2}$, are measured what is commonly known as the weather station method. The refractive index is then calculated by using equations from Edlen $(19 ; 20)$ or Ciddor (21). However the use of weather station data has many technical difficulties $(4 ; 22 ; 23)$ and various investigations have been performed to measure the refractive index using different methods (20).

The second method to determine the refractive index is with the use of a refractometer which measures the refractive index directly by comparing an optical path in air to the same optical path in a vacuum. Many studies have been undertaken in this area (25). Most of them focus on the verification of the weather station method, both for the Edlen and the Ciddor equation (22).

One of the most recognised works in this area is by Bonsch (22). The refractometer was designed for accurate measurements of the refractive index of gases under well-defined conditions. The measurements were performed for dry and most air using four wavelengths distributed over the visible part of the spectrum. Measured values were then compared with values determined by the modified Edlen's equation, using the weather station method. A fitting procedure was also described to adjust the humidity dependence and the constant term of the dispersion formula so that optimum agreement was obtained. A standard deviation of $3 * 10^{-9}$ for the residuals described the reproducibility of the measurements and the success of the fitting. For a $50 \% \mathrm{RH}$, a standard uncertainty of $1 * 10^{8}$ for the fitted formula is claimed, arising mainly from the uncertainty contributions of air parameters, temperature, pressure, relative humidity and $\mathrm{CO}_{2}$. The main objective of the paper was to verify the Edlen equation with special focus on the water vapour term 
in the equation. Although the results are extremely pleasing this system was designed for that specifically and not to be used in everyday measurements.

Ishige (21) described a system that measures the air refractive fluctuations using phase modulation homodyne interferometry. The method is based on a combination of a phase modulation homodyne laser interferometer, an external cavity laser diode and an ultra-low thermal expansion material. The interferometer used a Michelson interferometer which was constructed on the plate of ultra-low expansion material, so that the optical path change or the refractive index fluctuation caused by the thermal disturbance of the plate could be neglected. The diode laser was controlled by adjusting its frequency to track some of the dark fringes of the interferometer, so that the refractive index can be derived from the laser diode change. The uncertainty of the refractive index measurement in the setup was in the order of $1 * 10^{-8}$ and yet again although the results verify accuracy of the system, the measurement technique and apparatus is not suited to everyday use.

Zygo (22; 23) and Agilent (Hewlett- Packard Company, HP) (24) sell commercial systems which are optical wavelength compensators. Both took out patents in 1987 to protect their products which are strangely enough very similar in design. Figure 6 show the design of the Hewlett- Packard (Agilent) system. This system is a wavelength tracking compensator for a laser interferometer measurement system comprising a stable physical standard optical cavity and a double path, differential interferometer mounted on a baseplate. The interferometer monitors the apparent changes in the cavity's length caused by actual changes in the index of refraction of the surrounding air.

The Zygo system is an optical wavelength compensator which measured the change in refractive index of air. Since it measures only the relative change, it is important to know the index of refraction at the start of the measurement. This is achieved by using Edlen's equation and taking initial measurements of the temperature, pressure and relative humidity. Both systems employ the measurement and reference beams of the refractometer to travel across the same nominal distance, the reference beam through the vacuum tubes and the measuring beam through air. The difference between the two represents the change in the refractive index. Both of these system are only wavelength trackers and do not measure the absolute refractive index. 
U.S. Patent Aug. 23, $1988 \quad$ Sheet 1 of $5 \quad 4,765,741$

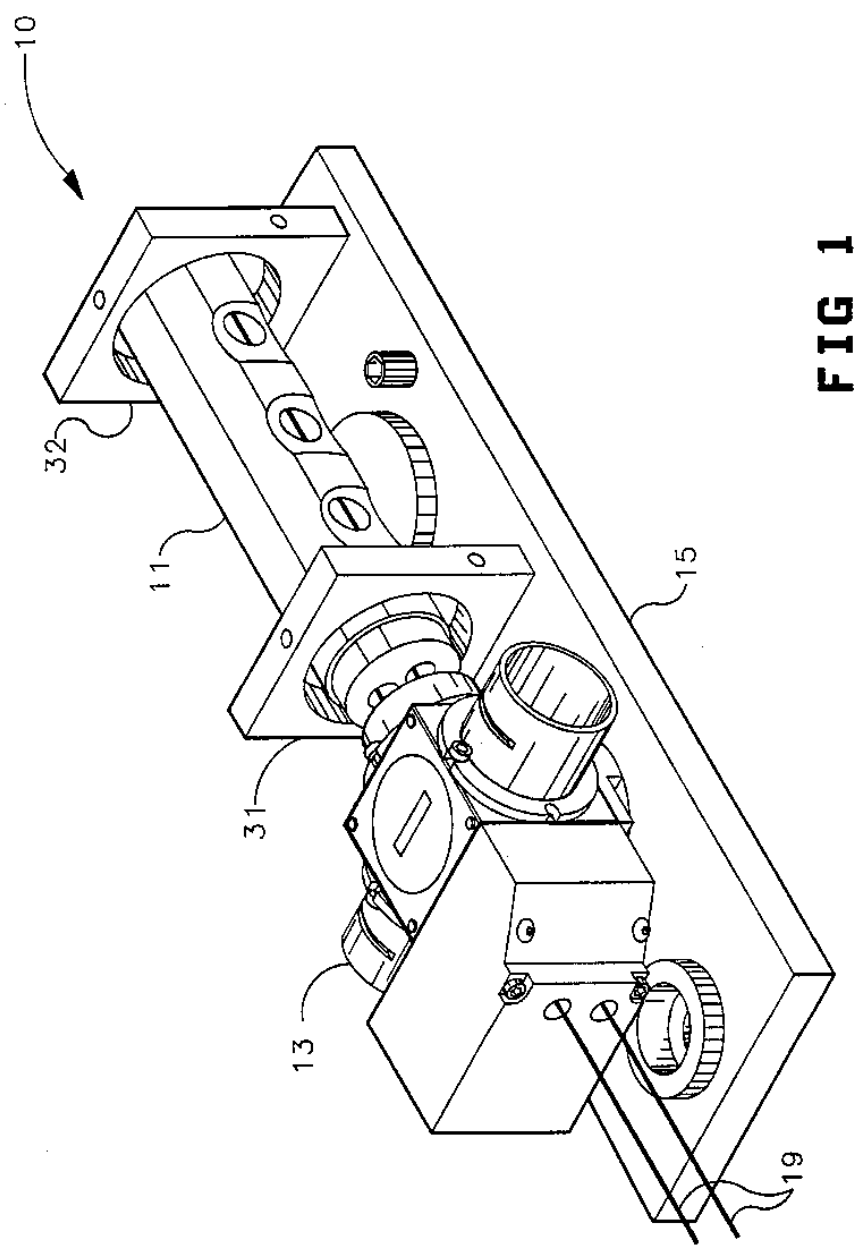

Figure 6: Patented Hewlett- Packard designed wavelength tracker. 
As can be seen, both of these commercial systems however only measure the fluctuation/variation in the air refractive index and not the absolute refractive index of air.

A similar system is described by Lazar $(25 ; 26)$ Therein he proposed an approach with two countermeasuring interferometers acting as a combination of both tracking and a displacement interferometer referencing the wavelength of the laser source to a mechanical standard made of a material with ultra-low thermal expansion. This technique combined length measurement within a specified range with measurement of the refractive index fluctuations in one axis. The method has been tested in comparison with the measurement of the refractive index of air in a thermally controlled environment to an accuracy of $5 * 10^{-8}$.

Meiners-Hagen (27) measured the refractive index by using two-colour interferometry. This is a well known method for measuring the refractive index of air and hence compensating for length based measurement. The speed of light is measured using two different wavelengths using the dispersion relation. No measurement of temperature, pressure and $\mathrm{CO}_{2}$ is necessary. However this method is valid only for dry air and is practically not used. For moist air the partial pressure of water vapour has to be known as the only air parameter for the compensation of the refractive index. In practice it is not possible to measure the partial pressure of water vapour in air directly. Standard hygrometers indicate the relative humidity only.

To realise the partial pressure the temperature has to be measured as well. Experiments were performed with this modified compensation method where the air pressure and humidity are measured conventionally. A homodyne interferometer was used with a frequency doubled $\mathrm{Nd}^{\mathrm{YVO}} \mathrm{V}_{4}$ laser with wavelengths of $1064 \mathrm{~nm}$ and $532 \mathrm{~nm}$. The effective temperature in the measurement path is derived from the length results for both wavelengths. The results show a length dependant uncertainty of below $1.2 * 10^{-7}$ for distances up to $30 \mathrm{~m}$.

This method measures the absolute refractive index but uses an expensive two colour method. Also as can be seen the water vapour term must be known which makes this method not ideal for everyday measurements. There are numerous other works that utilises refractometers indirectly to measure quantities used in refractive index calculations $(5 ; 6)$. Two PhD thesis' that of Ellis (32) and Lewis (33) used interferometry techniques extensively in their measurement designs. Ellis's thesis is "Optical metrology techniques for Dimensional stability measurement" describes interferometer techniques used to measure the stability of the sample for dimensional stability. Obviously in the interferometer measurements, refractive index measurements are required.

First a transmission Fabry-Perot cavity was used for both refractive index tracking and wavelength correcting interferometry. The refractive index tracking was measured to be within $4.9 * 10^{-8}$. In the wavelength correcting experiments, the difference between the weather station method and the wavelength corrected interferometer was approximately $2 \mathrm{~nm}$ over a $100 \mathrm{~nm}$ range. While a FabryPerot wavelength tracker could be used to correct for refractive index fluctuations, it requires a stable cavity made of thermally stable materials. Additionally, the Fabry-Perot mirrors must be highly efficient and they must be mounted in a thermally neutral plane. This increases the complexity and cost of the cavity design and more optical components are needed to utilize this system in conjunction with a sample measuring interferometer. Since this type of system employs a completely different measurement principle to measure and correct the refractive index, an alternative method was used to correct the refractive index fluctuations. 
An alternative method presented in the literature was to develop a dual interferometer. The schematic diagram is shown in Figure 7. A stabilised laser is used where the beam is split into two at the first beam splitter, BS1. The beam is further split into two beams again, given four beams, at the polarised beam splitter, PBS1. From here one interferometer beam measures the displacement of the sample while the other measures refractive index fluctuations as it directed through the vacuum cell. On the other side the beams are combined in the same way it was split, first a polarised beam splitter and then a normal beam splitter. The beams are directed onto photo diodes, PD which is used to measure the displacement of the sample and the vacuum cell.

One interferometer measures the sample fluctuations and the other measures refractive index fluctuations. If the refractive index fluctuations are nominally uniform throughout the interferometer, then this configuration could be suitable for measuring refractive index fluctuations. A detailed uncertainty analysis was presented and the two largest uncertainty contributors are the phase measurement itself, which in our case was not relevant as we used a different laser measuring system and the temperature fluctuation. This was also a problem in our design and will be discussed under the Initial Results in Chapter 6.1.

For the research in this thesis, a dual interferometer was chosen because the measurement and reference beams from both interferometers would pass through the same components, adding more balance into the system. Additionally, the sample measurement and refractive index measurement would both be performed with heterodyne interferometry. For the final design a dual interferometer system was used where the dimensional artefact tested for dimensional stability was directly compared to a vacuum cell. This system is similar in our final design but differs as it is designed to measure very small changes in length. The system could not be considered as it explains that it was designed to only measure length changes of $\mathrm{nm}$ with maximum changes of a few $\mu \mathrm{m}$.

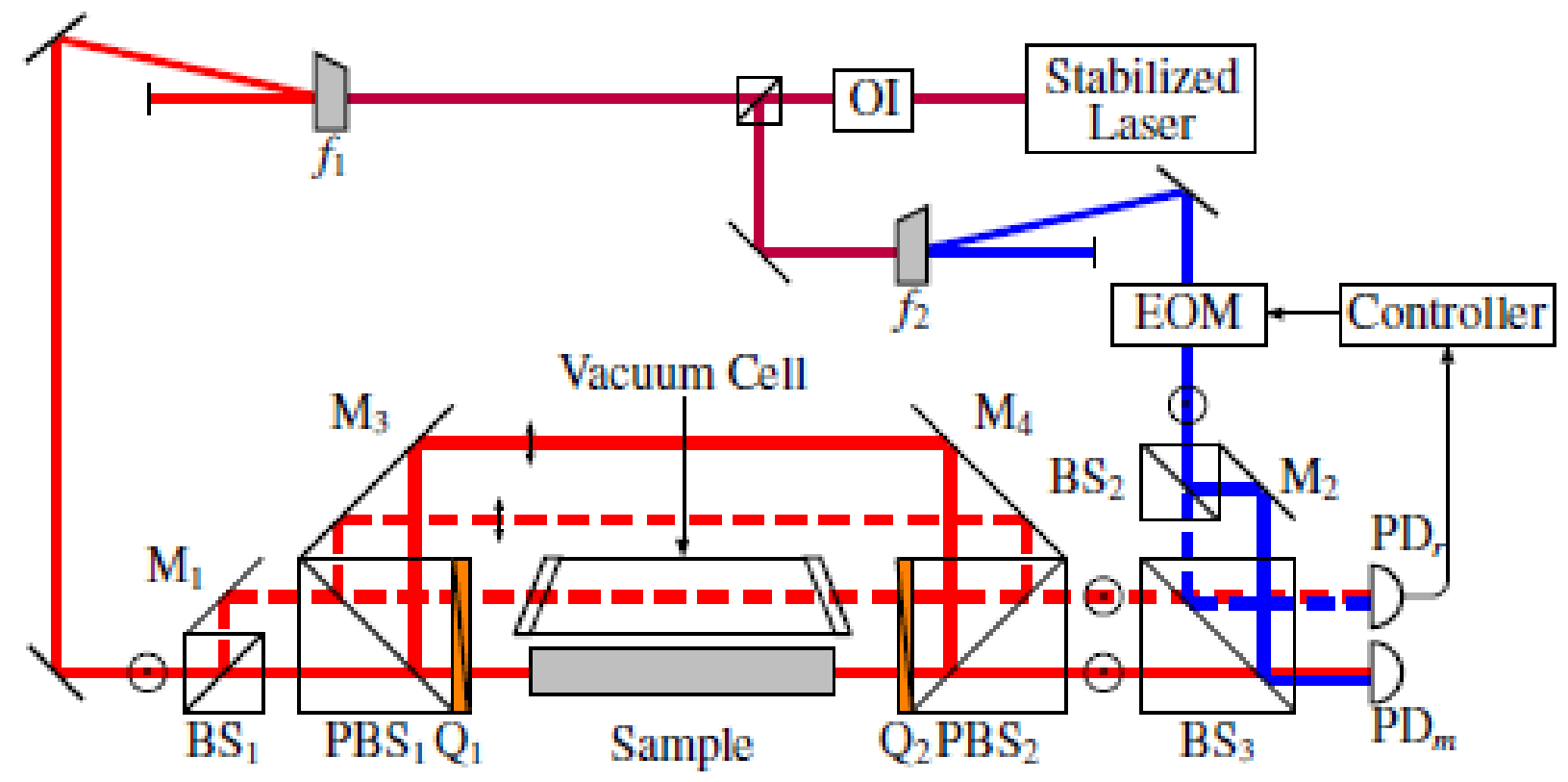

Figure 7: Schematic of the dual interferometer. 
Lewis's PhD is the "Absolute length measurements using multiple-wavelength phase-stepping interferometry". As it use interferometry to measure in this case gauge blocks and length bars, the measurement of the refractive index of air is very important. It uses a refractometer to validate the weather station method. The design of this system explained in the thesis is not for everyday use but only as a validation system (33). 


\section{Chapter 4: Theory}

\subsection{Edlen's equation}

The refractive index is as in Equation 1:

$$
n=\frac{c}{v^{\prime}}
$$

where $n$ is the refractive index and $c$ is the velocity of light in a vacuum and $v^{\prime}$ the velocity of light in a particular medium, in this case air (34).

The majority of laboratories use a weather station method to calculate the refractive index which measures air temperature, pressure and humidity and thereafter employs the Edlen equation.

Equation 2 is the original 1966 Edlen equation used to calculate the refractive index of air (19) .

$$
(n-1)_{T p}=\frac{p(n-1)_{s}}{720,775} * \frac{1+p(0,817-0,133 T) * 10^{-6}}{\left(1+0,0036610_{T}\right)} .
$$

Here $(n-1)$ is the refractivity of standard air, the temperature T is expressed in degrees Celcius, the air pressure $\mathrm{p}$ in torr, standard air at 1 atmosphere and $15^{\circ} \mathrm{C}$.

In 1988 revisions to the original Edlen equation was started by Muijlwijk (23) and furthered in 1992 by Beers (35) and in 1993 by Downs and Birch (20). These revisions look into subsequent improvements in data for the density of the air, refractivity of water vapour and changes in the temperature scale. The equation has also been changed to use only SI units and thus became (at standard conditions $\mathrm{T}=20^{\circ} \mathrm{C}, \mathrm{p}=100 \mathrm{kPa}, 0,04 \% \mathrm{CO}_{2}$ content):

$$
(n-1)_{T p}=\frac{p(n-1)_{S}}{93214,60} * \frac{1+10^{-8} p(0,5953-0,009876 T)}{\left(1+0,0036610_{T}\right)}
$$

The refractive index, $\eta_{t p}$, is for dry air. The refractive index for non-dry air, $\eta_{t p} \cup$, is calculated by:

$$
\eta_{t p} U-\eta_{t p}=-U *\left(3,7345-0,0401 \lambda^{2}\right) * 10^{-10}
$$

where the vapour pressure, $u$, is in pascals and $\lambda$ is the wavelength.

The Modified Edlen equation (3) is mainly used by metrologists in laboratories and by commercial 
manufacturers of laser measurements systems such as in Agilent (36), Zygo (37) and Renishaw (38). Hence we also decided to utilise the modified Edlen equation in this research. The equations of the different manufacturers are shown below, which although based on the modified Edlen equation, do have slight variations in them. These variables are discussed below.

The equation used by Agilent (36) for the refractive index calculation is:

$$
n=0,3836391 * \frac{1+10^{-6} p(0,817-0,133 T)}{(1+0,0036610 T)}-3,033 * 10^{-3} h\left(e^{-0,057627 T}\right),
$$

where $T$ is temperature, $p$ is pressure and $h$ is the relative humidity.

The equation used by Zygo (37) is:

$n=1+\left(3,8369 \cdot 10^{-7}\right) * \frac{1+p(0,817-0,133 T) \cdot 10^{-6}}{(1+0,0036610 T)}-5,6079433 \cdot 10^{-3} f$

where,

$$
f=\frac{h}{100} \cdot\left(4,07859739+0,44301857 * T+0,00232093 \cdot T^{-2}+0,00045785 \cdot T^{-3} .\right.
$$

and

$$
n=3,836391 \cdot p \frac{1+p \cdot(0,817-0,133 T) * 10^{-6}}{(1+0,0036610 T)}-3,033 * 10^{-3} \mathrm{~h}\left(e^{-0,057627 T}\right) .
$$

Eqs.(7) and (8) are the Edlen equations with a power series expansion for the water vapour pressure. Eq. (9) is an alternative using an exponential fit, where $T$ is temperature, $p$ is pressure and $h$ is the relative humidity.

The equation (9) used by Renishaw (38) is a more simplified equation when compared to the original modified Edlen equation:

$$
n=1+\left(\frac{7,86 e^{-5} * p}{273+T}\right)-1,5 e^{-11} * \mathrm{~h} *\left(T^{2}+160\right)
$$




\subsection{Ciddor's equation}

Another equation by Ciddor (17) was also developed with the conditions calculated for dry air at $15^{\circ} \mathrm{C}, 101,325 \mathrm{kPa}$ and $450 \mathrm{ppm}$ :

$$
\left(\eta_{T p}-1\right)=\left(\eta_{T p}-1\right) *\left[1+0,534 * 10^{-6}\left(x_{c}-450\right)\right] .
$$

Where $\eta_{t p}$ is the refractive index for dry air.

In most metrology applications, laser interferometers with a vacuum wavelength of nominal value $632,8 \mathrm{~nm}$ is used and measurements are performed close to the standard reference temperature of $20{ }^{\circ} \mathrm{C}(35)$. For these the Edlen equation is the preferred choice (36) over the Ciddor equation. Ciddor (17) claims his equation is slightly more accurate under extreme conditions and is therefore mainly used in surveying measuring equipment when long distance measurements are performed which ranges over kilometres and where the temperature is not controlled and has large fluctuations. 


\section{Chapter 5: Design of the Refractometry}

\subsection{Principles of length measurements using distance laser interferometers}

A Helium Neon gas laser produces a coherent light beam in the laser head. The two frequencies, $f 1$ and $\mathrm{f} 2$ differ by their opposite circular polarisations. From the laser tube, the beam containing both the frequencies passes through a quarter wave plate and a half wave plate which changes the circular polarisations to linear perpendicular polarisations. One being vertically, and the other horisontally polarised. Before the laser exits the laser head, the beam passes through a beam splitter where a small portion of the beam is sampled. This portion of the beam is used both to generate a reference frequency and to provide an error signal to the laser cavity tuning system. The difference in the amplitudes of $f 1$ and $f 2$ is used for tuning while the difference in frequency between $\mathrm{f} 1$ and $\mathrm{f} 2$ is used for the reference signal. The difference in frequency between $\mathrm{f} 1$ and $\mathrm{f} 2$ ranges anything from $1.5 \mathrm{MHz}$ (a Zeeman split laser system) to $20 \mathrm{MHz}$ for a polarization stabilised laser using acousto-optic modulator depending on the manufacturer (41).

Figure 8 shows a heterodyne laser system. The output beam from the dual frequency laser source contains two polarisations, one with a frequency $f 1$, and the other with frequency $f 2$. The beat frequency between them is $\mathrm{f} 2-\mathrm{f} 1$. A polarising beam-splitter reflects the light with frequency $\mathrm{f} 1$ into the reference path. Light with frequency $\mathrm{f} 2$ passes through the splitter into the measurement path where it reflects the moving reflector causing the frequency of the reflected beam to be Doppler shifted by $\delta F$. This reflected beam is then combined with the $f 1$ frequency light at the interferometer, and returned to the laser detector unit with a new beat frequency of $f 2-f 1 \pm \delta f$. Figure 9 shows how the beat frequency of a heterodyne laser system varies with the velocity of the moving reflector. When the optics are stationary, the beat frequency is $f 2-f 1$. As the optics move apart, the beat frequency rises by $\delta f$, if they move together it falls. High-resolution position sensing therefore requires stable measurement of the signal phase (41). 


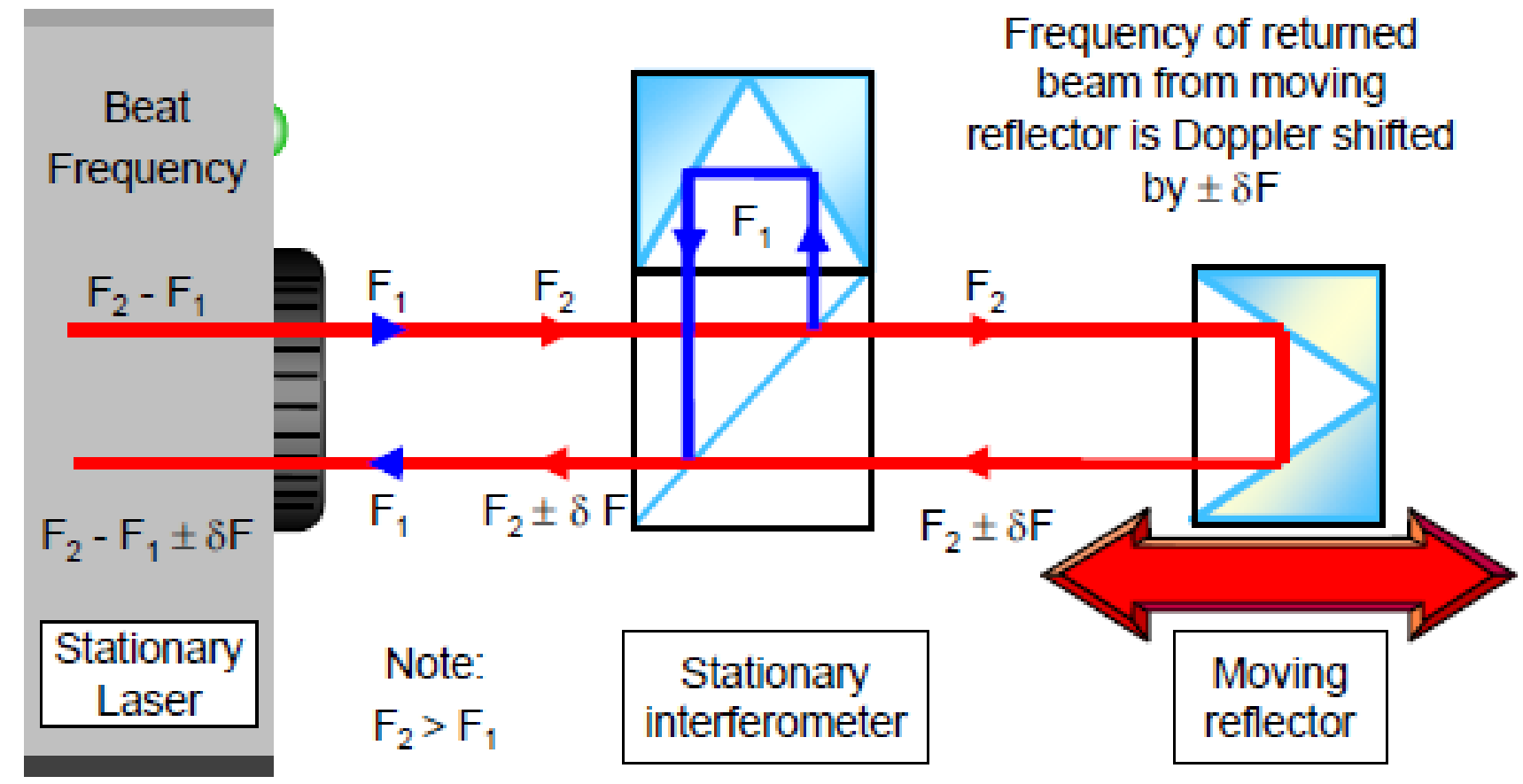

Figure 8: Operation of a heterodyne laser system (41).

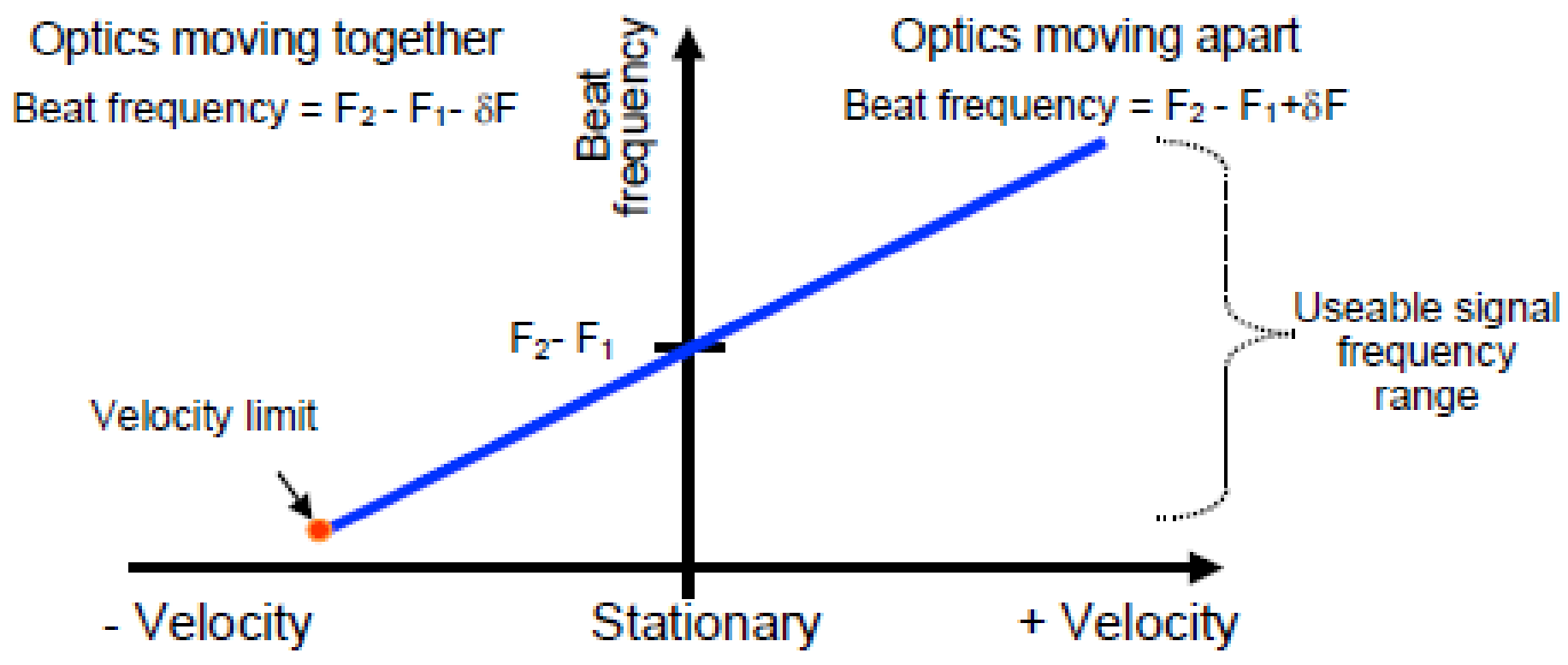

Figure 9: Heterodyne laser system, beat frequency versus velocity (41). 
The most common laser displacement lasers in metrology laboratories including NMISA are; Renishaw, Zygo, Sios and HP (now Agilent). Theoretically any of the above mentioned laser systems could be used but in the design a commercial dual frequency laser, Zygo ZMI 2000 interferometer was used. The Zygo system has the capability of operating two axes. This gives the opportunity to use one of the axes for the displacement measuring and the other for the index of refraction measurements. The frequency difference of the two laser beams is for the Zygo system about 20 $\mathrm{MHz}$ (37).

\subsection{Refractometer designs}

The design and use of a refractometer is not new and even before the metre was redefined as the speed of light traveled in a vacuum, Terrien (38) describes back in 1965 an air refractometer for interference length metrology. The refractometer for the measurement of the refractive index of the air is described in which two interference fringes are produced and used, one in air and the other in vacuum. The achieved accuracy aim for the design was $1 * 10^{-8}$ which was limited by the vacuum wavelength. The refractometer was placed inside an airtight isothermal enclosure and operated from the outside. The research into refractometers was highlighted as described in Chapter 2 where the metre was defined by the wavelength of Krypton in 1960.

US Patent, 5394244, (39) was filed in 1992 and describes the principles of a refractometer. This patent postulated an improved laser refractometer that employed an interferometer as the optical measuring portion, an object mirror and an etalon. The etalon is moved into the path of a plurality of reference beams and a plurality of measurement beams such that the reference beams travel through a vacuum within the etalon while the measurement beams travel a like distance through the etalon in ambient air. The etalon which is used as the vacuum is shown in Figure 7. 
$\begin{array}{llll}\text { U.S. Patent } & \text { Feb. 28, } 1995 & \text { Sheet } 2 \text { of } 4 & 5,394,244\end{array}$
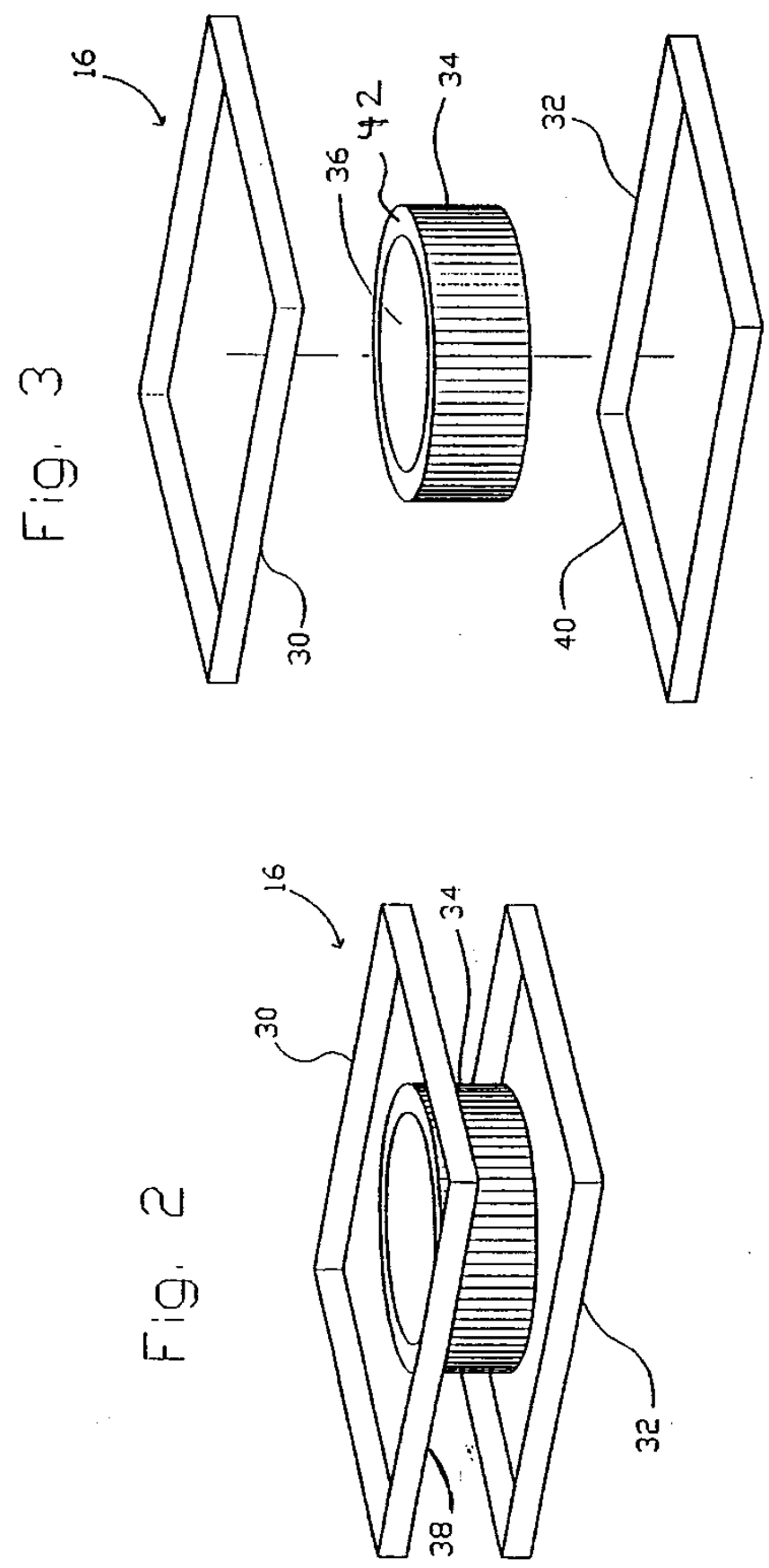

Figure 10: Patented refractometer showing the vacuum etalon which is moved through the laser beams. 
Recent work by Bonsch (22) in 1988 published research into the use of a refractometer to measure the refractive index of air. To perform the measurements in a refractometer, the laser interferometer is reset to zero when the air temperature, air pressure and humidity are stable. A vacuum is then drawn and the laser reading recorded. The change in the laser reading is due to the difference in path between the one frequency of the laser beam in the vacuum compared to the other frequency in air. This laser reading is then added to the tube length and divided by the tube length to calculate the refractive index of the air at that given time.

Schellekens (44) did a comparison of air refractometers in 1986, three years after the definition of the metre was changed. The paper describes the comparison of four refractometers designed and built in four Metrology (NMIs) laboratories. Direct comparison was realised by coupling the instruments to the same air inlet system. In order to compare the results the refractive index was also calculated from accurate measured values of temperature, pressure, relative humidity and $\mathrm{CO}_{2}$ content and using Edlen equation. The individual results show an agreement to within $1 * 10^{-7}$ while direct comparisons showed an agreement to within $5 * 10^{-8}$.

Research in the design of refractometers went far back with the first definition change of the metre to use wavelength and therefore requires accurate measurement of the refractive index. Recent research focuses mostly on the validation of the Edlen and Ciddor equations. The aim of this research was to design a cost effective, robust air refractometer which could be used in everyday measurements. This was also designed to be used in a permanent setup as part of a distance measurement system.

\subsection{Experimental refractometer set-up}

The formula in equation (11) for this calculation of $n$, the refractive index is by using $L$ the length of the tube and / the laser reading as described by Zygo (27):

$$
n=\left(\frac{L+l}{l}\right)
$$

A refractometer was designed to measure the velocity of light by directly comparing the same optical path of a laser beam in air to that in a vacuum. Figure 8 shows a schematic layout of the laser beams.

The refractometer was designed to use, as far as possible, commercial available components

Agilent angle interferometer, $10770 \mathrm{~A}$, optics is used to split the two laser beams in the polarising beam splitter and separated the two beams $\mathrm{f} 1$ and $\mathrm{f} 2$ to be directed parallel but a distance of 32,62 $\mathrm{mm}$ apart (36). The two laser beams are reflected by a large Agilent retroreflector back to the angle interferometer optics. A Zygo quarter wave plate was placed in front of the interferometer optics and used to change the polarisation of the laser beams to allow both beams to return to the laser detector and not pass straight through the polarisation beam splitter. 


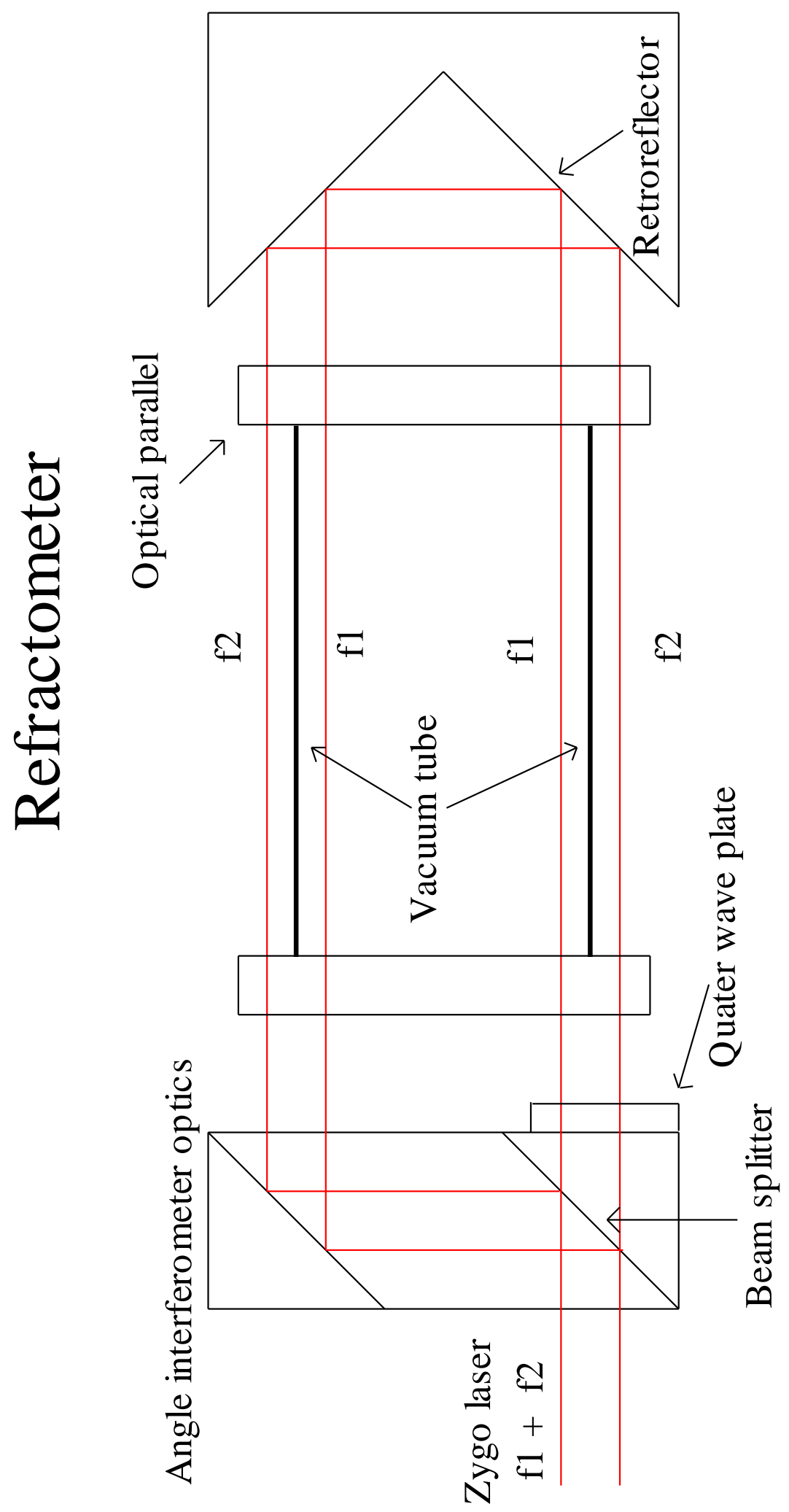

Figure 11: Schematic diagram of the designed Refractometer. 
Page | 32 


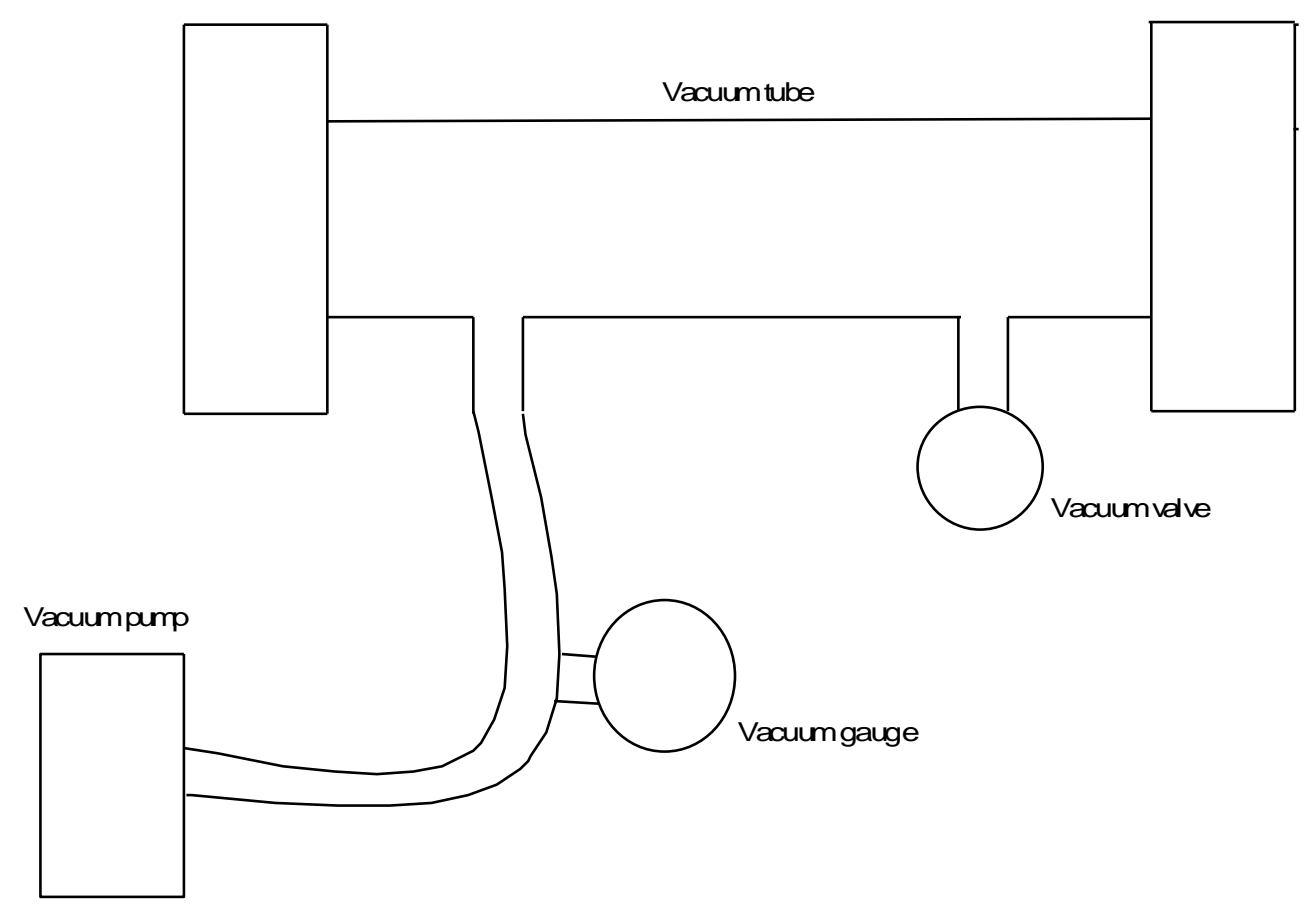

Figure. 12. Schematic diagram of the vacuum tube showing the layout of the vacuum pump and the gauge.

Two different vacuum layouts were tested for the use in this work.

Figure 11: show the original vacuum layout for a vacuum with two inlets. The inlet on the left had a vacuum pump connected to it, together with a vacuum gauge to measure the level of vacuum the pump had activated. To the inlet on the right of the vacuum tube, a vacuum valve was connected. This was used to control the level vacuum in the tube. The final vacuum layout will be discussed under the Chapter 6, Results.

The vacuum tube was manufactured from $3 \mathrm{~mm}$ Pyrex glass as it is a stable material with a good thermal conductivity to assure good transfer of the temperature inside and outside the vacuum cell (45). Other refractometer designs (22) used fused silica and although it is more stable and have a lower coefficient of thermal expansion than Pyrex glass it was felt for the design the high cost of fused silica cannot be justified. Under Chapter 7, Uncertainty calculations, the effect of using glass as the insulating material is discussed.

The tube is positioned in such a way that the one frequency of the laser; (f1) passes through the tube and the other frequency; ( $f 2$ ) passes through the air around the tube as shown in Figure 9 . The vacuum tube had two optical flats; $55 \mathrm{~mm}$ in diameter, attached on each end as is displayed in Figure 13. In order to eliminate any internal reflections from the surfaces, the optical flats were designed to be flat for minimum beam deviation but not parallel. For the optical flats, fused silica was use as it was deemed a critical component. Since the laser beam has to propagate through the optical flats, the material must not just be flat but also optically uniform.

The tube was manufactured with the two optical flats larger than the tube so that both the laser beams ( $f 1$ and $f 2$ ) pass through the same amount of optics and thus the only difference in optical 
length will be the vacuum-air path.

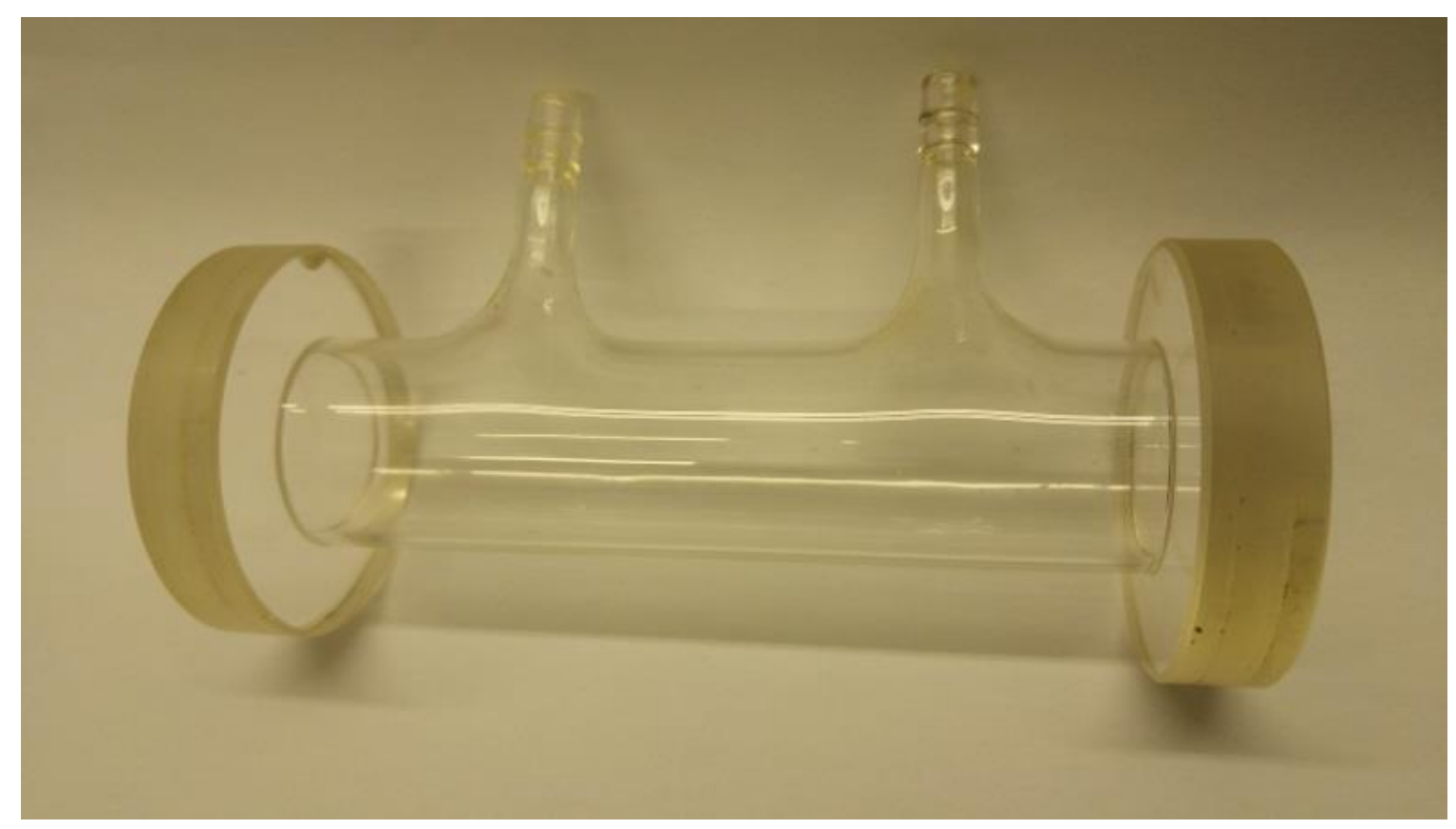

Figure 13: Vacuum tube with two optical flats bonded to the sides.

Before the system was tested with the vacuum, the stability of the optics was investigated without the vacuum tube. The results showed that even with large temperature fluctuations the laser readings stayed at zero to less than three nanometers which showed a stable optical setup. It was also found that the long-term stability, ie over a period of longer than a month, was more than adequate with the difference in the laser reading not changing by more than three nanometers, i.e. the same as the short term fluctuations. The effect of a few nanometers to the final refractive index measurements will be discussed under the uncertainty calculations. This improved optical stability is owed to the optical path lengths of the laser being equal for both frequencies and therefore identically affected. 


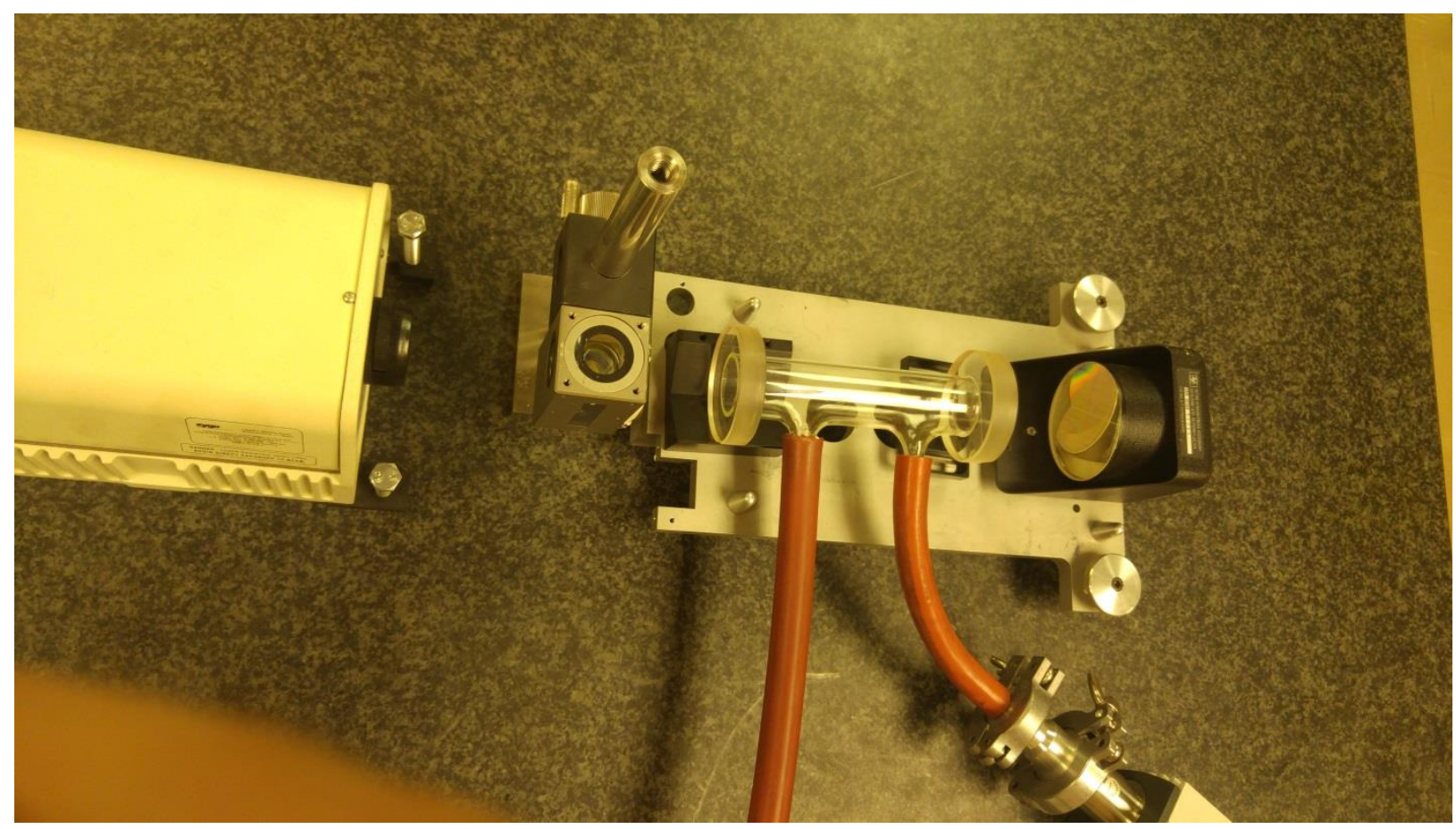

Figure 14: Picture of completed refractometer

The length of the tube was measured on a DEA Global Coordinate Measuring Machine before the optical flats were joined to the sides and once again after the optics were applied. The length of the tube was measured to be $120,231 \mathrm{~mm}$ to an accuracy of $5 \mu \mathrm{m}$, which is very conservative. The effect of this $5 \mu \mathrm{m}$ in length of the tube relative to the refractive index readings will be discussed under the Uncertainty Calculations in Chapter 7. It was found necessary to measure the tube under vacuum to determine if the length changed under vacuum. The results between air and when under vacuum were less than $2 \mu \mathrm{m}$ and it could not be established if this was due to the vacuum or repeatability as this was within the uncertainty of the measurements. The tube length value when under vacuum was used during the calculations. Studies have shown that the side widows, optical flats in this case, distort under vacuum $(22 ; 46 ; 47)$. However Bonsch $(22)$ calculated this distortion to be in the order of $7 \mathrm{~nm}$ only which is nearly 1000 times less than the uncertainty of the length of the tube measurements and thus negligible in the refractometer uncertainty calculations. 


\section{Chapter 6: Results}

\subsection{Initial Results}

The first readings did not exhibit substantial repeatability and were far out from what was expected. The expected refractive index was known as it was calculated using the weather station method. The difference in refractive index from the refractometer compared to the weather station was about double the value expected. The standard deviation from the first set of results was only around $1 \mathrm{ppm}$ and with the overall target of better than $1 * 10^{-7}$ these could not be used as reliable readings.

The non-repeating and unanticipated results led to investigation into all the possible measurement errors that could contribute to the poor results of unfavorable accuracy.

The investigation was focused around the following areas as these were the main sources that could lead to these unsatisfactory results:

i) general environmental conditions, and more specifically temperature fluctuations

ii) uneven vacuum

iii) tube length

iv) refractive index from refractometer calculation

v) laser measurements

In the design of measuring systems one of the first areas which must be under control is to make sure the system is repeating the measurements. Therefore the two areas which could influence the repeatability were the environmental conditions surrounding the refractometer and the required vacuum.

i) The refractometer was covered with a perspex cover to ensure better temperature stability and to restrict air flow over the refractometer. Perspex is generally used in a dimensional laboratory to improve temperature stability. This cover will not be used in the routine setup where the refractive index will be measured, but in the experimental setup to test the accuracy of the system. It was thus decided to cover the vacuum tube with the perspex cover.

ii) The next area of investigation was to ascertain if the vacuum was consistent and low enough to warrant acceptable readings. The investigation into the vacuum required is handled separately under 6.2. However to check if the vacuum is consistent the layout of the vacuum gauge was changed as in Figure 13. The final layout of the vacuum tube relative to the vacuum pump, gauge and valve is shown in Figure 13. The vacuum gauge and valve were swapped. The reason for this was that it was felt that in the layout as displayed in Figure 10, the vacuum gauge was measuring the level of vacuum in the pipe before the vacuum tube and not the vacuum in the tube itself. With the vacuum gauge installed on the other end of the vacuum tube it was felt that the gauge will more accurately measure the vacuum in the tube, or conservatively a higher vacuum.

iii) These improvements managed to fix the repeatability of the refractive index measurements but not the large offset. The other area which was investigated for the large offset was firstly the length of the tube. The length of the tube was measured using different techniques but because of the readings being so far away from the expected values, a 
normal Vernier caliper was used to verify that the length measured on the DEA CMM was correct. The readings were within $20 \mu \mathrm{m}$ which is with the uncertainty of the Vernier calliper. This value will not be used for the final calculation but was to see if there is a large error which could contribute to the large deviation from the expected value. The tube length was verified to $20 \mu \mathrm{m}$ and could not be the cause for the unanticipated readings.

iv) The calculation was checked and although the equation used was from the Zygo manual, the equation was verified by the equation used by the other research in refractometers. The equation used by all the refractometers was the same even if they used different laser, interferometer measuring techniques.

v) The last area which was then different from the other refractometers was the laser measurement system. The optical layout was different from the other refractometer designs and also different from the original Zygo laser measurement setups. The laser measuring techniques had to be investigated as describe in 5.1, wherefater the problem was solved.

After these areas was corrected the readings was as expected and reported in 6.3.

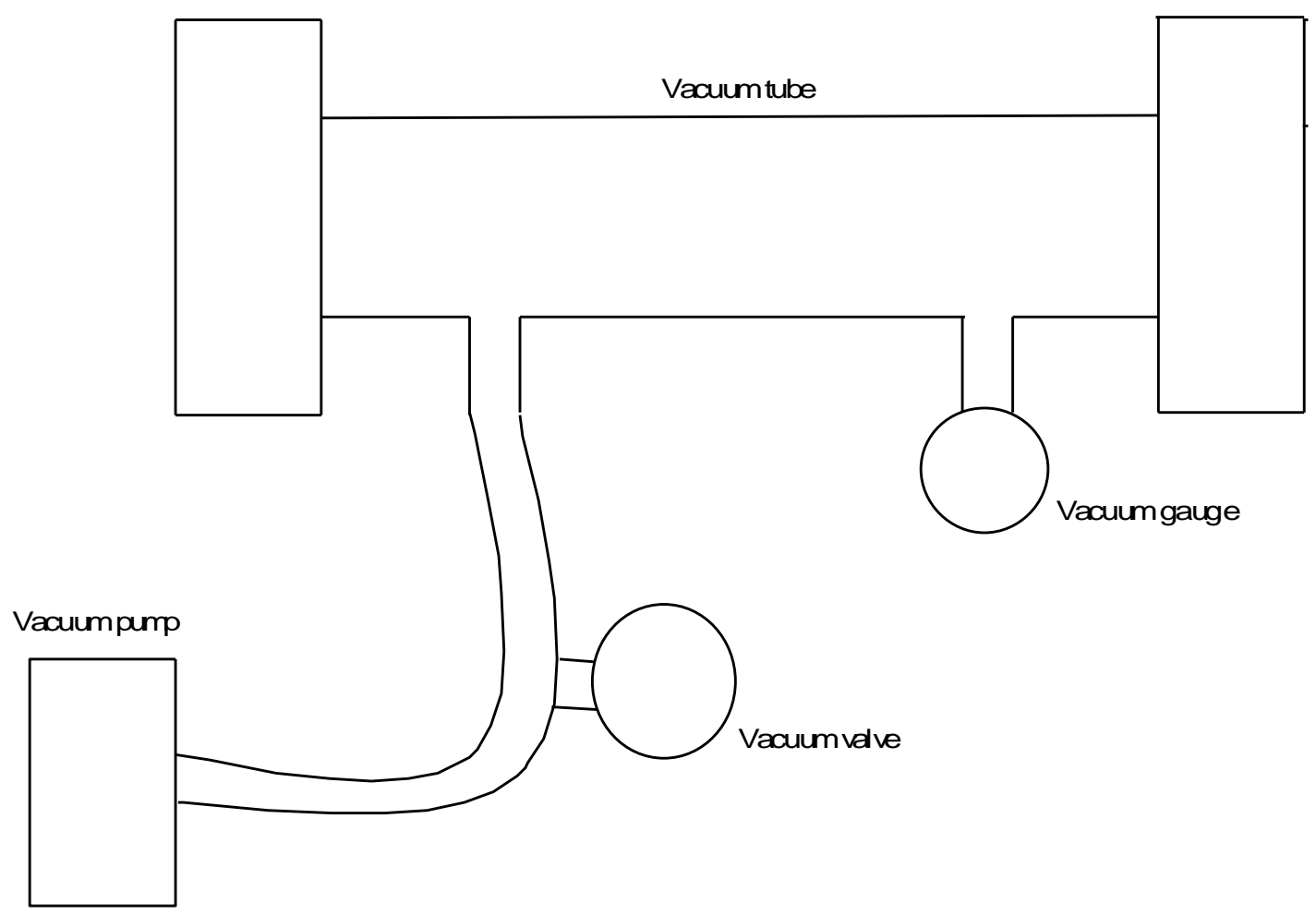

Figure 15: Vacuum tube with layout changed to move the vacuum tube to the other inlet of the vacuum tube. 


\subsection{Determination of vacuum required}

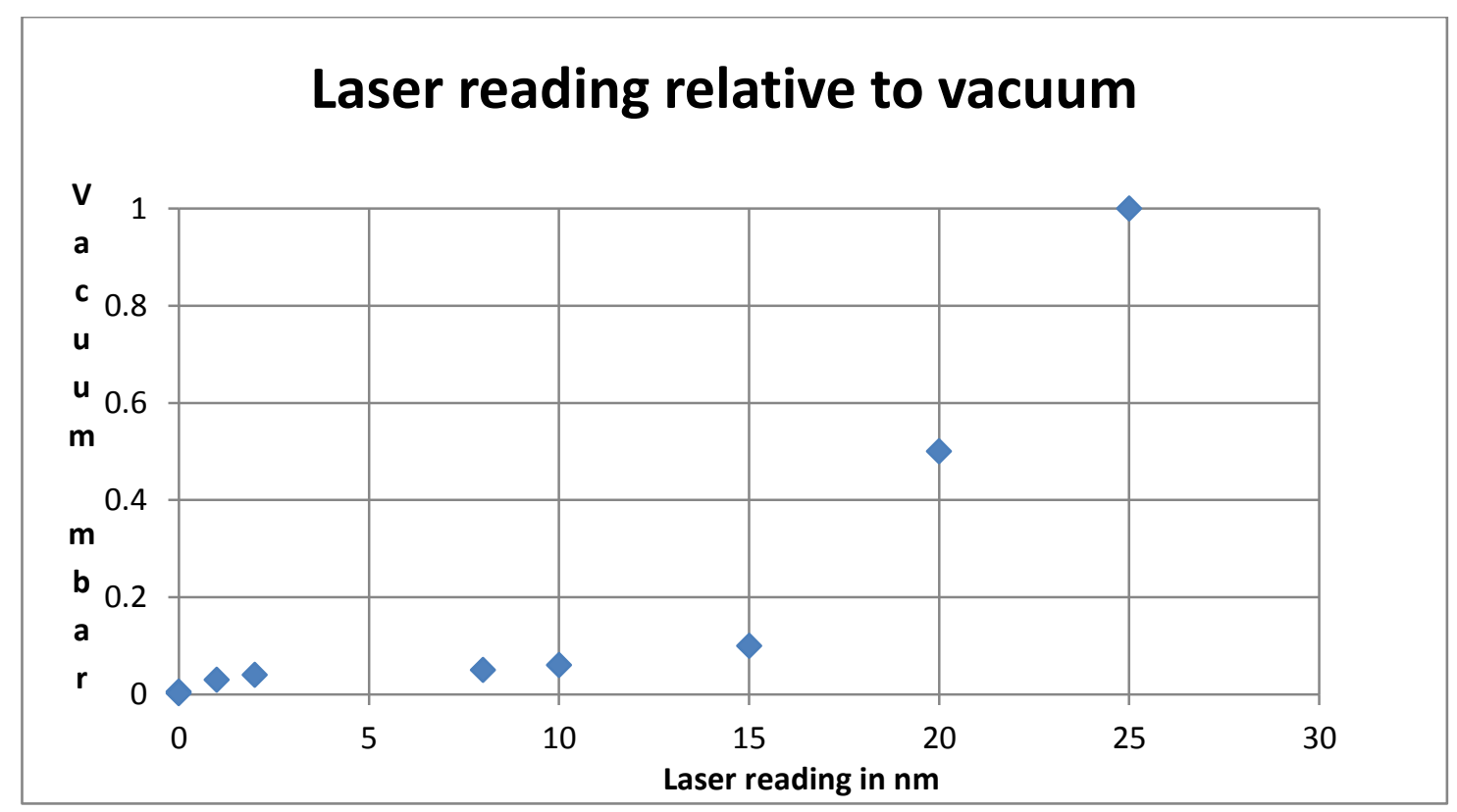

Graph 1. The laser reading measured relative to different levels of vacuum.

An investigation was performed to establish which level of vacuum is required to measure the refractive index accurately. Bonsch (18) indicated a pressure of $1 * 10^{-2} \mathrm{~Pa}$. In this investigation mbar was used. Although the SI unit for pressure is Pascal (44), in the measurement of atmospheric pressure, mbar is still the most used unit. The conversion from $\mathrm{Pa}$ to mbar is according to the SI unit $1 \mathrm{~Pa}=0,01 \mathrm{mbar}(44)$. The value in mbar that Bonsch (18) proposed is then $1 * 10^{-4} \mathrm{mbar}$. The lowest vacuum achieved was only $1 * 10^{-3}$. This is 10 times higher than what was suggested by Bonsch which led to this investigation.

The laser was reset to zero at the lowest vacuum achieved. Thereafter the valve was slowly opened, controlled and the laser reading relative to the vacuum recorded. The laser reading relative to the vacuum is plotted in Graph 1. The readings show that as the vacuum reduces (higher pressures), the laser reading increases. The readings show that even at $1 * 10^{-2}$ mbar the laser reading display only a few nanometers which is the uncertainty of the laser reading. Therefore a vacuum of $1 * 10^{-3} \mathrm{mbar}$ is sufficient to measure the refractive index using this refractometer. 


\subsection{Final Results}

Although the refractive index was calculated, it had to be validated as by itself there is no means to test if the calculations are correct. For this validation the refractive index from the refractometer was calculated and compared to refractive indices calculated using the weather station method. Accurate temperature sensors, an air pressure barometer and humidity sensor were used to measure the various air conditions and these were then implemented in the Edlen equation calculation. The calculated refractive index was then compared to the refractive index measured by the refractometer.

\begin{tabular}{||l|l|l|l|l|l|l|l||}
\hline \hline $\begin{array}{l}\text { Reading } \\
\text { Number }\end{array}$ & $\begin{array}{l}\text { Laser } \\
\text { reading in } \\
\text { vacuum } \\
(\boldsymbol{\mu m})\end{array}$ & $\begin{array}{l}\text { Air } \\
\text { temperature } \\
\left({ }^{\circ} \mathbf{C}\right)\end{array}$ & $\begin{array}{l}\text { Air } \\
\text { Pressure } \\
\text { (mbar) }\end{array}$ & $\begin{array}{l}\text { Air } \\
\text { Humidity } \\
(\% \mathrm{RH})\end{array}$ & $\begin{array}{l}\text { Refractive index } \\
\text { from } \\
\text { refractometer }\end{array}$ & $\begin{array}{l}\text { Refractive } \\
\text { index from } \\
\text { weather station } \\
\text { (Edlen) }\end{array}$ & $\begin{array}{l}\text { Difference } \\
\text { between } \\
\text { Refractometer } \\
\text { and Edlen }\end{array}$ \\
\hline $\mathbf{1}$ & 28,038 & 19,899 & 870,51 & 45,2 & 1,000233201 & 1,000233197 & $-4,08791 \mathrm{E}-09$ \\
\hline $\mathbf{2}$ & 28,022 & 19,912 & 870,06 & 42,5 & 1,000233068 & 1,000233089 & $2,09893 \mathrm{E}-08$ \\
\hline $\mathbf{3}$ & 27,932 & 20,019 & 867,76 & 47 & 1,000232319 & 1,000232398 & $7,85483 \mathrm{E}-08$ \\
\hline $\mathbf{4}$ & 27,811 & 19,943 & 863,32 & 42 & 1,000231313 & 1,000231259 & $-5,40557 \mathrm{E}-08$ \\
\hline $\mathbf{5}$ & 27,873 & 19,847 & 863,54 & 39,9 & 1,000231882 & 1,000231951 & $6,85133 \mathrm{E}-08$ \\
\hline $\mathbf{6}$ & 28,055 & 19,651 & 870,57 & 40,6 & 1,000233397 & 1,000233456 & $5,90562 \mathrm{E}-08$ \\
\hline $\mathbf{7}$ & 28,034 & 19,725 & 870,17 & 40,9 & 1,000233222 & 1,000233285 & $6,28015 \mathrm{E}-08$ \\
\hline $\mathbf{8}$ & 28,055 & 19,775 & 870,27 & 40,1 & 1,000233342 & 1,000233278 & $-6,44824 \mathrm{E}-08$ \\
\hline $\mathbf{9}$ & 28,058 & 19,827 & 870,76 & 41,1 & 1,000233367 & 1,000233401 & $3,35656 \mathrm{E}-08$ \\
\hline $\mathbf{1 0}$ & 28,015 & 19,992 & 869,68 & 38,9 & 1,000233010 & 1,000232952 & $-5,77895 \mathrm{E}-08$ \\
\hline
\end{tabular}

Table 1: Results for the refractometer data compared to the modified Edlen equation using weather station measurement calculations.

Table 1 shows some of the results with different environmental conditions, validating the results over these conditions. The refractometer results were compared only with those obtained from the Edlen weather station method. The last column is the difference between the two methods, Edlen and refractometer. An average difference between the two methods was calculated to be $1.4 * 10^{-8}$. This is in very good agreement considering that the modified Edlen equation is, according to the references $(16 ; 17 ; 18)$, only accurate to $3 * 10^{-8}$ without taking into account the accuracy of the sensors.

Stone describes a method (41) where helium is used as a standard for correcting any errors in a gas refractometer. It describes that the refractive index of helium at atmospheric pressure can be accurately determined. Therefore, helium can serve as a theory based standard of refractive index and can be used as a medium of known refractive index for high accuracy interferometric length measurements. It can also be used to correct for errors in gas refractometers. The system was validated by measuring the refractive index of nitrogen using two refractometers and the results agreed to with-in a few parts in $10^{9}$. This method requires more investigation and can be considered in future research.

These readings were taken over a 12 month period to test if the readings were comparable at 
different environmental conditions and long term repeatability. The results show that over the period there was no significant difference that could be linked to the change in environmental conditions. The results are captured in the paper by the authors Chetty and Kruger (43).

\subsection{Investigation of temperature, pressure and humidity fluctuations}

In previous paragraphs it is explained that the agreement between the refractometer and the Edlen equation, weather station method agree to $1,4 * 10^{-8}$. However, the graphs were analysed in a way to establish if there is a trend in the temperature, pressure or humidity fluctuations relative to the refractive index. The differences in air temperature, pressure and humidity were plotted relative to the differences between the Refractive index measurements calculated form the Refractometer and the Edlen weather station method.

In all the graphs the environmental conditions, temperature, pressure and humidity plotted from low to high, relative to the refractive index calculated as in Table 1.

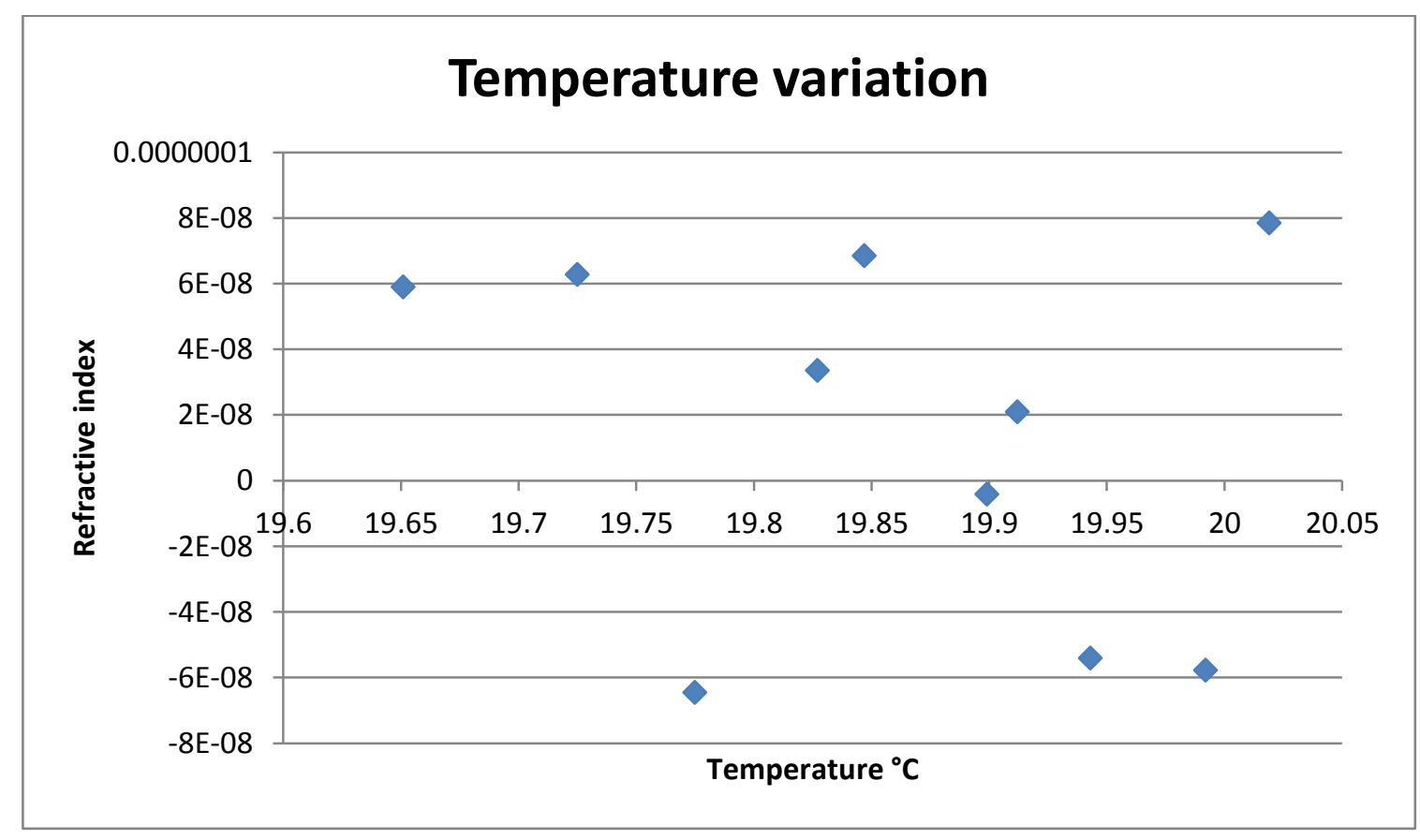

Graph 2: The refractive index plotted relative to the air temperature arranged from low to high. 


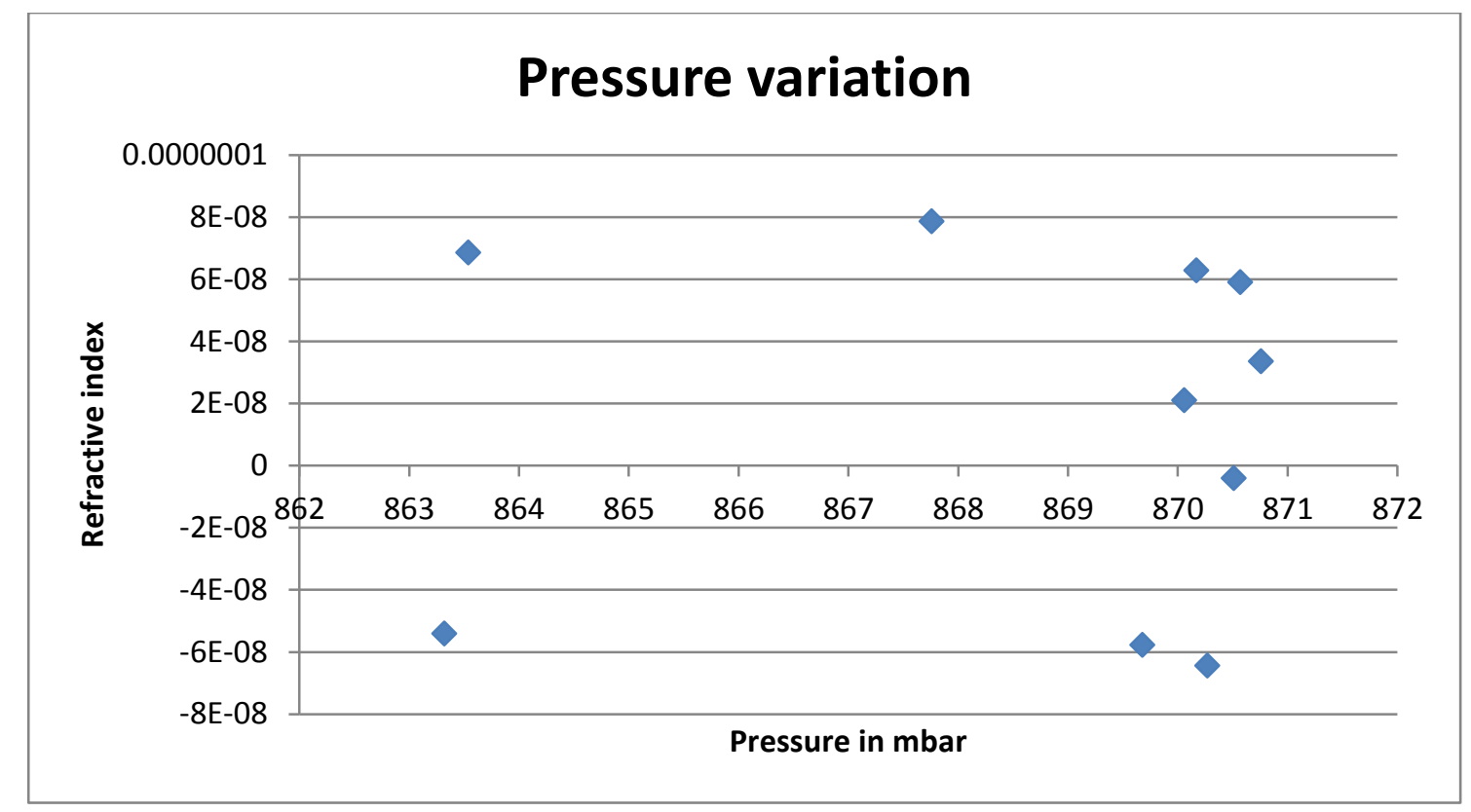

Graph 3: The refractive index plotted relative to the air pressure arranged from low to high.

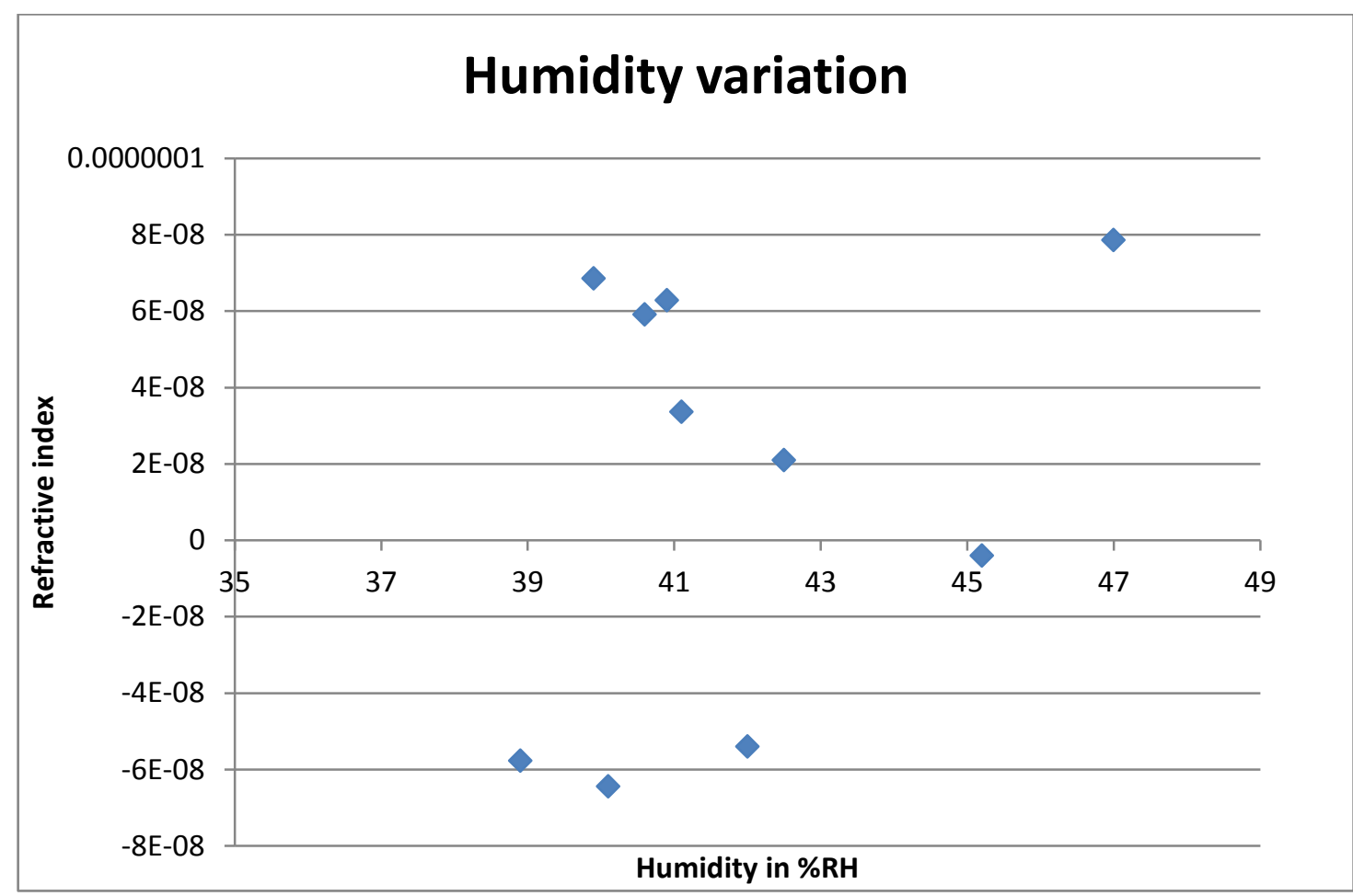

Graph 4: The refractive index plotted relative to the air relative humidity arranged from low to high.

The graphs were analysed to determine if there was a trend in the differences in refractive index measurements relative to the air temperature, the air pressure or air humidity. The results show a random spread which reflects that there is no systematic error introduced by performing the refractive index measurements over the operating range. 


\subsection{Comparison of refractometer results to commercial systems}

During the experiments a comparison was performed between the different methods for calculating the refractive index of air. One set of readings was calculated using the different systems. Readings of actual systems were used. Table 2 is the results of one set of readings taken by the refractometer compared to different calculation methods. The results show very good agreement, with Ciddor and Edlen providing exactly the same reading, the Agilent reading being accurate to $2 * 10^{-9}$. Unfortunately the Zygo and Renishaw software only displayed 7 digits after the decimal, but agreed up to this digit.

\begin{tabular}{|c|c|c|c|c|c|c||}
\hline $\begin{array}{c}\text { Refractive } \\
\text { index }\end{array}$ & Refractometer & Edlen & Ciddor & $\begin{array}{c}\text { Renishaw } \\
\text { (ver. 20.02) }\end{array}$ & $\begin{array}{c}\text { Zygo } \\
\text { (ver 2.2.4) }\end{array}$ & $\begin{array}{c}\text { Agilent } \\
\text { (ver. 2.003) }\end{array}$ \\
\hline Value & 1,00023301 & 1,000232952 & 1,000232952 & 1,0002329 & 1,0002329 & 1,000232954 \\
\hline
\end{tabular}

Table 2: Results of the last reading taken by the refractometer compared to different calculation methods.

\section{Chapter 7: Uncertainty calculations}

\subsection{Refractive index uncertainty of the Edlen weather station method}

In the calculation of the uncertainty for the refractometer, the GUM (50) was as far as possible used as the guideline.

The uncertainty for the Edlen weather station method was calculated by estimating the uncertainty in the measurement for each sensor and substituting the value into the Edlen equation to see the differences in the refractive index. The uncertainty of the measurements was taken not only from the uncertainty of the calibration, but also accounting for the accuracy of the measurements by the sensors during the measurements. This is necessitated by the fact that the sensors are calibrated under near ideal conditions but yet the measurements are performed in laboratory conditions. The uncertainties were estimated on the conservative side.

Almost all significant errors in determining the index of refraction does not come from uncertainty in the equation but from the inaccuracy in measurement of the atmospheric environment, mostly the air temperature and pressure. Measurement errors that could produce an error of $1 * 10^{-6}$ (1 part per million) in the index of refraction (hence a length-proportional error of $1 * 10^{-6}$ ) include:

- an error of $1^{\circ} \mathrm{C}$ in air temperature

- an error of $0.4 \mathrm{kPa}(3 \mathrm{~mm} \mathrm{Hg})$ in air pressure 
- an error of $50 \%$ in relative humidity at sufficiently high air temperatures

The uncertainty of the air temperature measurements was estimated to be $0,05^{\circ} \mathrm{C}$ which results in an uncertainty of $5,1^{*} 10^{-8}$ in the refractive index. The air humidity uncertainty was estimated to be $2 \%$ $\mathrm{RH}$ resulting in a $1,7^{*} 10^{-8}$ refractive index uncertainty and lastly the air pressure uncertainty was estimated to be $0,1 \mathrm{mbar}$ and this resulted in an uncertainty of $2,7 * 10^{-8}$ in the refractive index. If combining these uncertainties, using Root Sum Square, the overall uncertainties from the sensors is $5,9 * 10^{-8}$ in the refractive index.

The refractometer's simple design makes the uncertainty estimations less complicated when compared to the complex calculations for the weather station method. For this refractometer the only uncertainty contributors are; i) the length of the tube and ii) the accuracy of the laser reading.

\subsection{Refractive index uncertainty due to the length of vacuum tube}

The length of the tube was measured on a highly accurate coordinate measuring machine with a conservative accuracy of $2 \mu \mathrm{m}$.

$$
\mathrm{L}_{\text {tube } 20}=\mathrm{L}_{\text {tube }}+\left(\mathrm{L}_{\text {tube }} * \mathrm{CTE}_{\mathrm{L}} * \Delta \mathrm{t}\right)
$$

The effect of the temperature of the length of the tube was calculated to establish if it will be necessary to perform temperature correction during the refractive index measurements. The references temperature for dimensional measurement which is $20^{\circ} \mathrm{C}(36)$, was used as the temperature to perform the measurements.

Equation (12) was used to calculate the effect of the $5^{\circ} \mathrm{C}$ in the length uncertainty of the vacuum tube.

$\mathrm{L}_{\text {tube20 }}$ is the length of the vacuum tube at $20^{\circ} \mathrm{C}$. $\mathrm{L}_{\text {tube }}$ is the length of the tube at the measured temperature.

$\mathrm{CTE}_{\mathrm{L}}$ is the co-efficient of linear expansion of glass, taken as $8 * 10^{-6} \mathrm{~m} / \mathrm{m} *{ }^{\circ} \mathrm{C}(45 ; 46 ; 47)$, and $\Delta \mathrm{t}$ the temperature difference from the reference temperature. From the results in Table 1 the temperature difference was $0,6^{\circ} \mathrm{C}$.

The $L_{\text {tubezo }}$ is calculated to be $0,6 \mu \mathrm{m}$, which is less than the uncertainty of the $2 \mu \mathrm{m}$ accuracy to which the tube length was measured and therefore no corrections to the length due to temperature fluctuations will be necessary.

\subsection{Refractive index uncertainty due to laser reading}

The uncertainty attributed from the laser system is calculated following the Zygo laser manual. According to the manual the following contributors must be considered: 
However from the simple design of the refractometer, only a few contributors are relevant to this setup. Each is explained according to the ZYGO manual setup for a Zygo displacement laser.

- Temperature variation, Measuring in refractometer.

- Pressure variation, Measured in refractometer.

- Humidity variation, Measured in refractometer.

- Range of motion, Only a $25 \mu \mathrm{m}$ range measured, the difference between the laser readings of the vacuum and air.

- Dead path distance, none, Same for both measuring paths, vacuum and air.

- Interferometer dead path, Same for both measuring paths, vacuum and air.

- Thermal coefficient of artifact, covered under 7.2.

- Laser stability, better than 0,01 ppm (parts per million).

- Electronics accuracy, 1,6 $\mathrm{nm}$.

- Polarization mixing $2 \mathrm{~nm}$.

- Target mirror angle, Same for both measuring value, vacuum and air

- Abbé offset, Same for both measuring value, vacuum and air

- Target mirror flatness, Same for both measuring value, vacuum and air

Following the ZYGO method the total Root Sum Square total of the uncertainty attributed from the laser measuring system for this setup will be $2,6 \mathrm{~nm}$ only.

\subsection{Conclusions and recommendation from Uncertainty calculations.}

The uncertainty of the Edlen weather station method was calculated to be $5,9 * 10^{-8}$. The refractometer uncertainty was calculated to be $1,8 * 10^{-8}$. This is thus an improvement of 3 times over the theoretical calculation of the Edlen weather station method.

This results in an uncertainty of less than $1^{*} 10^{-8}$ in the refractive index. The uncertainty of the laser reading is taken as $2,6 \mathrm{~nm}$ which results in a $1,5^{*} 10^{-8}$ uncertainty in the refractive index. The combined uncertainty for the refractometer is then close to $1,8 * 10^{-8}$.

One uncertainty contributor which may require further investigation is the zero reset position. As described under the experimental setup, the laser reading is zero and at the start of the measurement where after the vacuum is drawn. This is however assuming that the air temperature inside and outside the vacuum tube is the same. A perspex cover was used to keep the air temperature around the tube stable and good stabilisation time was allowed, but potentially a temperature difference can still exist. It is very difficult to measure the air temperature accurately, especially inside the vacuum tube and this could not be performed. However the cover was used to reduce this effect. 


\title{
Chapter 8: Journal paper
}

The paper in this Chapter has been published after stringent peer review in Applied Optics, volume 55, issue 32 in November 2016.

\begin{tabular}{l}
\hline 9118 vol. 55, No. 32/ / November 102016 / Appofied optics \\
Robust air refractometer for accurate \\
compensation of the refractive index of \\
air in everyday use
\end{tabular}

\section{O. Kruger AND N. ChettY*}

School of Chemistry and Physics, Discipline of Physics, University of KwaZulu-Natal (PMB), Private Bag X01, Scottsville 3209, South Africa "Corresponding author: ChettyN3@ukzn.ac.za

Received 1 June 2016; revised 4 October 2016; accepted 4 October 2016; posted 4 October 2016 (Doc. ID 265034); published 7 November 2016

\begin{abstract}
The definition of the meter is based on the speed of light in a vacuum; however, most dimensional measurements, when performed using laser interferometry, are performed in air. A velocity of light compensation needs to be applied to the velocity of the laser light for accurate measurements of the speed of light to be approximated in a vacuum. Most practices use a weather-station method, whereby the ambient conditions are measured. Thereafter, the modified Edlén's equation is used, and corrections are calculated for the wavelength of the laser. The theoretical calculation is, however, only accurate to $3 * 10^{-8}$ without taking into account the accuracy of the sensors. Thus, this work focuses on investigations into the velocity of light compensations, both to improve upon the accuracy of the Edlén equation method in everyday use, and to verify the accuracy of the current weather-station systems in use through comparison with the refractometer. A refractometer that allows for velocity of light compensation measurements was developed, tested, and verified. The system was designed to be simple and costeffective for use in everyday dimensional measurements, but with high accuracy. Achieved results show that although simple in design, the refractometer is accurate to at least $1 * 10^{-8}$, which meets our initial condition for design. (ㅇ 2016 Optical Society of America
\end{abstract}

OCIS codes: (120.3180) Interferometry; (120.3930) Metrological instrumentation; (120.3940) Metrology.

http://dx.doi.org/10.1364/AO.55.009118

\section{INTRODUCTION}

The 1889 definition of the meter, based on the international prototype of platinum-iridium, was replaced by the 11 th General Conference on Weights and Measures (CGPM) in 1960 , using a definition based on the wavelength of krypton 86 radiation. This change was adopted in order to improve the accuracy to which the definition of the meter could be realized. The realization was achieved using an interferometer with a travelling microscope to measure the optical path difference as the fringes were counted. In turn, this was replaced in 1983 by the 17th CGPM. The meter has been, since 1983, defined as "the length of path traveled by light in vacuum during a time interval of $1 / 299792458$ of a second" [1-3].

The need for accurate length measurements and, subsequently, a definition, is needed throughout the modern world as captured in the strategy of the Consultative Committee for Length [4]. Much of industry and technology relies on these measurements. With dimensional metrology's ever-increasing accuracy, it necessitates relooking at the uncertainty contributors to dimensional measurements.
The meter is defined as the speed of light in a vacuum. However, most of the dimensional and length measurements are performed in air. The refractive index in air differs with different environmental conditions (air pressure, air temperature, humidity, and $\mathrm{CO}_{2}$ ), and the refractive index of air at specific conditions has to be calculated to make corrections to the speed of light in air, compared to the speed of light in a vacuum.

To make this correction, the refractive index of air at specific environmental conditions must be calculated. To calculate the refractive index, the speed (velocity) of light in a vacuum $c$, is divided by $v^{\prime}$, the velocity of light in air at the time the measurements are taken.

There are two methods to determine the refractive index. In the first method, the environmental conditions, air temperature, air pressure, relative humidity, and $\mathrm{CO}_{2}$, are measured. The refractive index is then calculated by using equations from Edlén $[5,6]$ or Ciddor [7]. The second method to determine the refractive index is with the use of a refractometer, which measures the refractive index directly by comparing an optical path in air to the same optical path in a vacuum, as described by Bonsch [8]. 
The use of weather-station data has many technical difficulties $[4,8,9]$, and various investigations have been performed to measure the refractive index using different methods [10-13]. Ishige [13] and Zygo [14] measure the air refractive fluctuations using phase modulation homodyne interferometry and an optical wavelength compensator. Lazar $[11,12]$ reduced the effect of refractive index variations by using two countermeasuring interferometers and material with ultralow thermal expansion. Meiners-Hagen [15] measured the refractive index by using two-color interferometry. The first method only measures refractive index fluctuations, and not the absolute refractive index. The second method directly measures the absolute refractive index but uses an expensive two-color method. There are numerous other works that utilize refractometers indirectly to measure quantities used in refractive index calculations [15-19].

The aim of this work was to design, build, and test a simple, robust, and cost-effective refractometer to be used in everyday laser-based measurements.

\section{THEORY AND REVISED EDLÉN'S EQUATION}

The refractive index is as in Eq. (1),

$$
n=\frac{c}{v^{\prime}} \text {, }
$$

where $n$ is the refractive index and $c$ is the velocity of light in a vacuum and $v^{\prime}$ the velocity of light in a particular medium, in this case air [19].

The majority of laboratories use a weather-station method, which measures air temperature, pressure, and humidity, and thereafter employs the Edlén equation to calculate the refractive index.

Equation (2) is the original 1966 Edlén equation used to calculate the refractive index of air [5]

$$
(n-1)_{T_{P}}=\frac{p(n-1)_{s}}{720.775} * \frac{1+p(0.817-0.133 T) * 10^{-6}}{\left(1+0.0036610_{T}\right)} .
$$

Here $(n-1)$ is the refractivity of standard air, the temperature $T$ is expressed in degrees Celsius, the air pressure $p$ in Torr, standard air at 1 atmosphere and $15^{\circ} \mathrm{C}$.

In 1988, revisions to the original Edlén equation were started by Muijlwijk and furthered in 1992 by Beers and Doiron [20], and in 1993 by Birch [5,6]. These revisions look into subsequent improvements in the data of the density of the air, refractivity of water vapor, and change in the temperature scale. The equation has also been changed to use only SI units, and thus became (at standard conditions $T=20^{\circ} \mathrm{C}$, $p=100 \mathrm{kPa}, 0.04 \% \mathrm{CO}_{2}$ content) [6]

$$
(n-1)_{T_{P}}=\frac{p(n-1)_{s}}{93214.60} * \frac{1+10^{-8} p(0.5953-0.009876 T)}{\left(1+0.0036610_{T}\right)} \text {. }
$$

The refractive index, $\eta_{t p}$, is for dry air. The refractive index for nondry air, $\eta_{t p} v$, is calculated by

$$
\eta_{t p} v-\eta_{t p}=-v *\left(3.7345-0.0401 \lambda^{2}\right) * 10^{-10},
$$

where the vapor pressure, $v$, is in pascals and $\lambda$ is the wavelength.

Another equation by Ciddor [7] was also developed with the conditions calculated for dry air at $15^{\circ} \mathrm{C}, 101.325 \mathrm{kPa}$, and 450 ppm:

$$
\left(\eta_{T_{P}}-1\right)=\left(\eta_{T_{P}}-1\right) *\left[1+0.534 * 10^{-6}\left(x_{c}-450\right)\right] .
$$

The modified Edlén equation (6) is mainly used by metrologists in laboratories and by commercial manufacturers of laser measurements systems, such as in Agilent [21], Zygo [22], and Renishaw [23]. Hence, we also decided to utilize the modified Edlén equation in this research. The equations of the different manufacturers are shown below, which although based on the modified Edlén equation, do have slight variations in them.

The equation used by Agilent [21] for the refractive index calculation is

$$
\begin{aligned}
n= & 0.3836391 * \frac{1+10^{-6} p(0.817-0.133 T)}{(1+0.0036610 T)}-3.033 \\
& * 10^{-3} h\left(e^{-0.057627 T}\right),
\end{aligned}
$$

where $T$ is temperature, $p$ is pressure, and $h$ is the relative humidity.

The equation used by Zygo [22] is

$$
\begin{aligned}
n= & 1+\left(3.8369 .10^{-7}\right) * \frac{1+p(0.817-0.133 T) * 10^{-6}}{(1+0.0036610 T)} \\
& -5.6079433 .10^{-3} f,
\end{aligned}
$$

where

$$
\begin{aligned}
f= & \frac{h}{100} \cdot(4.07859739+0.44301857 * T \\
& +0.00232093 . T^{-2}+0.00045785 . T^{-3},
\end{aligned}
$$

and

$$
\begin{aligned}
n= & 3.836391 \cdot p \frac{1+p \cdot(0.817-0.133 T) * 10^{-6}}{(1+0.0036610 T)} \\
& -3.033 * 10^{-3} h\left(e^{-0.057627 T}\right) .
\end{aligned}
$$

Equations (7) and (8) are the Edlén equations with a power series expansion for the water vapor pressure. Equation (9) is an alternative using an exponential fit, where $T$ is temperature, $p$ is pressure, and $h$ is the relative humidity.

Equation (10), used by Renishaw [23], is a more simplified equation when compared to the original modified Edlén equation:

$$
n=1+\left(\frac{7.86 e^{-5} * p}{273+T}\right)-1.5 e^{-11} * h *\left(T^{2}+160\right) .
$$

In most metrology applications, laser interferometers with a vacuum wavelength of nominal value $632.8 \mathrm{~nm}$ is used and measurements are performed close to the standard reference temperature of $20^{\circ} \mathrm{C}$. For these, the Edlén equation is the preferred choice [20] over the Ciddor equation. Ciddor [6] claims his equation is slightly more accurate under extreme conditions and is therefore mainly used in surveying measuring equipment when long distance measurements are performed that range over kilometers, and where the temperature is not controlled 
with large fluctuations. This view is supported by other reviews [24].

\section{REFRACTOMETRY}

The design and use of a refractometer is not new and a U.S. Patent, 5,394,244, "Ambient air refractive index measuring device" [25], was filed in 1992. Recent work by Bonsch [8] published research into the use of a refractometer to measure the refractive index of air. To perform the measurements in a refractometer, the laser interferometer is reset to zero when the air temperature, air pressure, and humidity are stable. A vacuum is then drawn and the laser reading recorded. The change in the laser reading is due to the difference in path between the one frequency of the laser beam in the vacuum compared to the other frequency in air. This laser reading is then added to the tube length and divided by the tube length to calculate the refractive index of the air at that given time. The formula (11) for this calculation is [22], where $n$ is the refractive index, $L$ the length of the tube, and $l$ the laser reading:

$$
n=\left(\frac{L+l}{l}\right) \text {. }
$$

\section{EXPERIMENTAL DETAILS}

A refractometer was designed to measure the velocity of light by directly comparing the same optical path of a laser beam in air to that in a vacuum. Figure 1 shows a schematic layout of the laser beams. Any laser system could theoretically be used, but in this design a commercial dual-frequency laser, Zygo ZMI 2000 interferometer was used. The frequency difference of the two laser beams is about $20 \mathrm{MHz}$ [22]. An Agilent angle interferometer, $10770 \mathrm{~A}$, optics is used to split the two laser beams in the polarizing beam splitter and separates the two beams, $\mathrm{fl}$ and $\mathrm{f} 2$, to be directed parallel but a distance of $32.62 \mathrm{~mm}$ apart [21]. The two laser beams are reflected by a large Agilent retroreflector back to the angle interferometer optics. A Zygo quarter-wave plate was placed in front of the interferometer optics and used to change the polarization of the laser beams to allow both beams to return to the laser detector and not pass straight through the polarization beam splitter.

The vacuum tube was manufactured and positioned in such a way that the one frequency of the laser (f1), passes through the tube and the other frequency (f2), passes through the air around the tube, as shown in Fig. 1. The vacuum tube is made up of a $3 \mathrm{~mm}$ glass tube with two optical flats, $55 \mathrm{~mm}$ in diameter, on each end, as displayed in Fig. 2. In order to eliminate any internal reflections from the surfaces, the optical flats were designed to be flat for minimum beam deviation but not parallel. The tube was manufactured with the two optical flats larger than the tube so that both the laser beams ( $\mathrm{fl}$ and $\mathrm{f2}$ ) pass through the same amount of optics and the only difference in optical length will be the vacuum-air path.

Before the system was tested with the vacuum, the stability of the optics was investigated without the vacuum tube. The results showed that even with large temperature fluctuations, the laser readings stayed at zero to less than $3 \mathrm{~nm}$, which showed a stable optical setup. It was also found that the

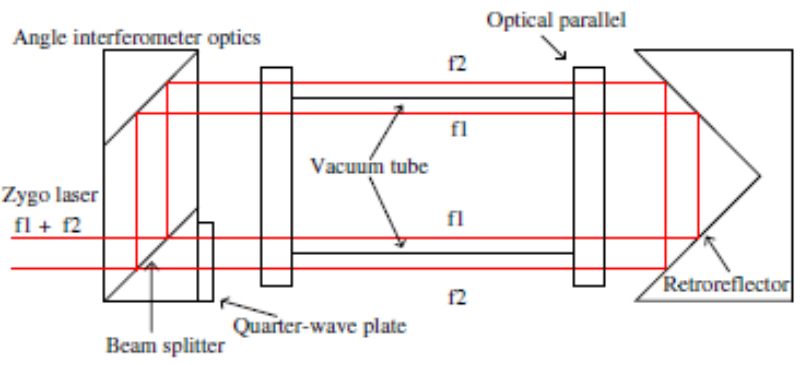

Fig. 1. Schematic diagram of proposed refractometer.

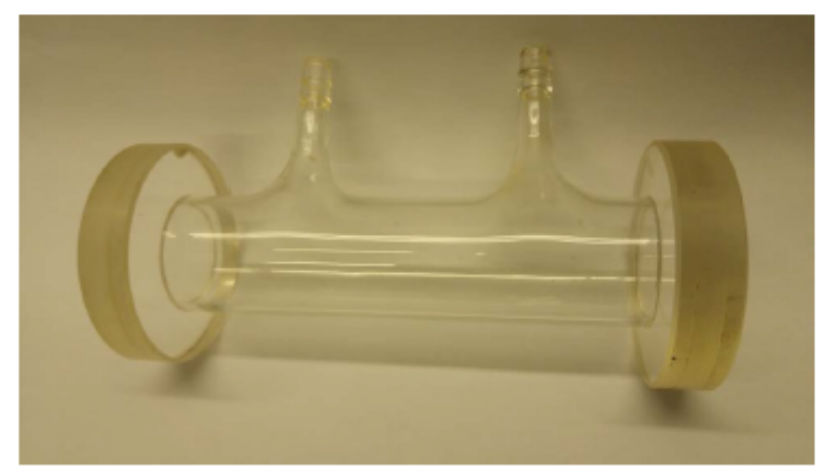

Fig. 2 Vacuum tube with two optical flats bonded to the sides.

long-term stability (i.e., over a period of longer than a month) was more than adequate, with the difference in the laser reading not changing more than $3 \mathrm{~nm}$ (i.e., the same as the short-term fluctuations). The effect of a few nanometers to the final refractive index measurements will be discussed under the uncertainty calculations. This improved optical stability is owed to the optical path lengths of the laser being equal for both frequencies and are therefore identically affected.

The length of the tube was measured on a DEA Global Coordinate Measuring Machine before the optical flats were joined to the sides and once again after the optics were applied. The length of the tube was measured to be $120.231 \mathrm{~mm}$ to an accuracy of $5 \mu \mathrm{m}$, which is very conservative. The effect of this $5 \mu \mathrm{m}$ will be discussed under the uncertainty section. It was found necessary to measure the tube under vacuum to determine if the length changed under vacuum. The results between air and when under vacuum were less than $2 \mu \mathrm{m}$, and it could not be established if this was due to the vacuum or repeatability, as this was within the uncertainty of the measurements. The tube length value when under vacuum was used during the calculations. Studies have shown that the side windows, optical flats in this case, distort under vacuum [8,26,27]. However, Bonsch [8] calculated this distortion to be in the order of $7 \mathrm{~nm}$ only, which is nearly 1000 times less than the uncertainty of the tube length measurements and negligible in the refractometer uncertainty calculations.

Lastly, the refractometer was covered with a perspex cover to ensure better temperature stability and to restrict air flow over the refractometer. 


\section{RESULTS}

Although the refractive index was calculated, it had to be validated, as by itself there is no means to test if the calculations are correct. For this validation, the refractive index from the refractometer was calculated and compared to refractive indices calculated using the weather-station method. Accurate temperature sensors, an air pressure barometer, and humidity sensor were used to measure the various air conditions, and these were implemented in the Edlén equation. The refractive index calculated was then compared to the refractive index measured by the refractometer.

Table 1 shows some of the results with different environmental conditions, validating the results over these conditions. The refractometer was compared only with those obtained from the Edlén weather-station method. The last column is the difference between the two methods, Edlén and refractometer. An average difference between the two methods was calculated to be $1.4 * 10^{-8}$. This is very good agreement, considering that the modified Edlén equation is, according to the references $[5,6-8]$, only accurate to $3 * 10^{-8}$ without taking into account the accuracy of the sensors.

These readings were taken over a six-month period to test if the readings were comparable at different environmental conditions and long-term repeatability. The results show that over the period, there was no significant difference that could be linked to the change in environmental conditions.

During the experiments, a comparison was performed between the different methods for calculating the refractive index of air. One set of readings was calculated using the different systems. Readings of actual systems were used. Table 2 shows the results of one set of readings taken by the refractometer compared to different calculation methods. The results show very good agreement, with Ciddor and Edlén providing exactly the same reading, the Agilent reading being to $2 * 10^{-9}$.
Unfortunately, the Zygo and Renishaw software only displayed seven digits after the decimal, but agreed up to this digit.

\section{UNCERTAINTY CALCULATIONS}

The uncertainty for the Edlén weather-station method was calculated by estimating the uncertainty in the measurement for each sensor and substituting the value into the Edlén equation to see the differences in the refractive index. The uncertainty of the measurements was taken not only from the uncertainty of the calibration, but also accounting for the accuracy of the measurements by the sensors during the measurements. This is necessitated by the fact that the sensors are calibrated under near ideal conditions, but yet, the measurements are performed in laboratory conditions. The uncertainties were estimated on the conservative side.

The uncertainty of the air temperature measurements was estimated to be $0.05^{\circ} \mathrm{C}$, which results in an uncertainty of $5.1 * 10^{-8}$ in the refractive index. The air humidity uncertainty was estimated to be $2 \% \mathrm{RH}$, resulting in a $1.7 * 10^{-8}$ refractive index uncertainty, and lastly, the air pressure uncertainty was estimated to be $0.1 \mathrm{mbar}$, and this resulted in an uncertainty of $2.7 * 10^{-8}$ in the refractive index. If combining these uncertainties, using root sum square, the overall uncertainties from the sensors is $5.9 * 10^{-8}$ in the refractive index.

The refractometer's simple design makes the uncertainty estimations less complicated when compared to the complex calculations for the weather-station method. For this refractometer, the only uncertainty contributors are: (i) the length of the tube and (ii) the accuracy of the laser reading.

The length of the tube was measured on a highly accurate coordinate measuring machine with a conservative accuracy of $2 \mu \mathrm{m}$, including for the thermal expansion coefficient of the glass over a temperature range of $5^{\circ} \mathrm{C}$. This results in an

Table 1. Results for the Refractometer Data Compared to the Modified Edlén Equation Using Weather-Station Measurement Calculations

\begin{tabular}{|c|c|c|c|c|c|c|c|}
\hline $\begin{array}{l}\text { Reading } \\
\text { Number }\end{array}$ & $\begin{array}{c}\text { Laser Reading } \\
\text { in Vacuum } \\
(\mu \mathrm{m})\end{array}$ & $\begin{array}{c}\text { Air } \\
\text { Temperature } \\
\left({ }^{\circ} \mathrm{C}\right)\end{array}$ & $\begin{array}{c}\text { Air } \\
\text { Pressure } \\
\text { (mbar) }\end{array}$ & $\begin{array}{c}\text { Air } \\
\text { Humidity } \\
(\% \mathrm{RH})\end{array}$ & $\begin{array}{c}\text { Refractive } \\
\text { Index from } \\
\text { Refractometer }\end{array}$ & $\begin{array}{l}\text { Refractive Index } \\
\text { from Weather } \\
\text { Station (Edlén) }\end{array}$ & $\begin{array}{c}\text { Difference Between } \\
\text { Refractometer } \\
\text { and Edlén }\end{array}$ \\
\hline 1 & 28.038 & 19.899 & 870.51 & 45.2 & 1.000233201 & 1.000233197 & $-4.08791 \mathrm{E}-09$ \\
\hline 2 & 28.022 & 19.912 & 870.06 & 42.5 & 1.000233068 & 1.000233089 & $2.09893 E-08$ \\
\hline 3 & 27.932 & 20.019 & 867.76 & 47 & 1.000232319 & 1.000232398 & $7.85483 \mathrm{E}-08$ \\
\hline 4 & 27.811 & 19.943 & 863.32 & 42 & 1.000231313 & 1.000231259 & $-5.40557 \mathrm{E}-08$ \\
\hline 5 & 27.873 & 19.847 & 863.54 & 39.9 & 1.000231882 & 1.000231951 & $6.85133 E-08$ \\
\hline 6 & 28.055 & 19.651 & 870.57 & 40.6 & 1.000233397 & 1.000233456 & $5.90562 \mathrm{E}-08$ \\
\hline 7 & 28.034 & 19.725 & 870.17 & 40.9 & 1.000233222 & 1.000233285 & $6.28015 E-08$ \\
\hline 8 & 28.055 & 19.775 & 870.27 & 40.1 & 1.000233342 & 1.000233278 & $-6.44824 \mathrm{E}-08$ \\
\hline 9 & 28.058 & 19.827 & 870.76 & 41.1 & 1.000233367 & 1.000233401 & $3.35656 \mathrm{E}-08$ \\
\hline 10 & 28.015 & 19.992 & 869.68 & 38.9 & 1.000233010 & 1.000232952 & $-5.77895 \mathrm{E}-08$ \\
\hline
\end{tabular}

Table 2. Results of the Last Reading Taken by the Refractometer Compared to Different Calculation Methods

\begin{tabular}{lcccccc}
\hline Refractive index & Refractometer & Edlén [5] & Ciddor [7] & $\begin{array}{c}\text { Renishaw [23] } \\
\text { (ver. 20.02) }\end{array}$ & $\begin{array}{c}\text { Zygo [22] } \\
\text { (ver 2.2.4) }\end{array}$ & $\begin{array}{c}\text { Agilent [21] } \\
\text { (ver. 2.003) }\end{array}$ \\
\hline Value & 1.00023301 & 1.000232952 & 1.000232952 & 1.0002329 & 1.0002329 & 1.000232954 \\
\hline
\end{tabular}


uncertainty of less than $1 * 10^{-8}$ in the refractive index. The uncertainty of the laser reading is taken as $2 \mathrm{~nm}$, which results in a $1.5 * 10^{-8}$ uncertainty in the refractive index. The combined uncertainty for the refractometer is then close to $1.8 * 10^{-8}$.

The uncertainty of the Edlén weather-station method was calculated to be $5.9 * 10^{-8}$. The refractometer uncertainty was calculated to be $1.8 * 10^{-8}$. This is thus an improvement of three times over the theoretical calculation of the Edlén weather-station method.

\section{CONCLUSION}

One advantage of the current developed refractometer is the simplicity of the design. From the discussions around the uncertainty contributions, it is clear that the system has very few uncertainty contributors. Furthermore, these do not have large contributions to the overall uncertainty. The system proves that a simple design with mainly commercial optics can be used to measure the refractive index to an accuracy of $1 * 10^{-8}$,

Lastly, it can be used in everyday measurements to the required accuracy in place of using the weather-station method. One disadvantage is the use of the vacuum pump during the measurements, where it generates heat, which is not ideal in dimensional measurements.

Further work will be to investigate a system that eliminates the use of a vacuum pump for the measurements. This would further simplify the method and allow for faster and slightly more accurate measurements.

\section{REFERENCES}

1. P. Giacomo, "Resolution 1," Metrologia, 20, 25-30 (1984).

2. T. J. Quinn, "Mise en Practique of the Definition of the metre," Metrologia 30, 523-541 (1994).

3. R. Felder, "Practical realization of the definition of the metre," Metrologia 42, 323-325 (1997).

4. BIPM, Strategy 2013-2023, Consultative Committee for Length.

5. B. Edlén, "The refractive index of air," Metrologia 2, 71-80 (1965).

6. K. P. Birch and M. J. Downs, "An updated Edlén equation for the refractive index of air," Metrologia 30, 155-162 (1993).

7. P.E. Ciddor, "Refractive index of air: new equations for the visible and near infrared," Appl. Opt. 35, 1566-1573 (1996)
8. G. Bonsch and E. Potulski, "Measurement of the refractive index of air and comparison with the modified Edlén's formulae," Metrologia 35, 133-139 (1998).

9. H. Haitjema, "Achieving traceability and sub-nanometer uncertainty using interferometric techniques," Meas. Sci. Technol. 19, 084002 (2008).

10. R. Muijilwijk, Update of the Edlén formulae for the refractive index of air," Metrologia 25, 189 (1988).

11. J. Lazar, "Suppression of air refractive index variations in highresolution interferometry," Sensors 11, 7644-7655 (2011).

12. J. Lazar, "Refractive index compensation in over-determined interferometric systems," Sensors 12, 14084-14094 (2012).

13. M. Ishige, "Measurement of air refractive index fluctuation from frequency change using a phase modulation homodyne interferometer and an external cavity laser diode," Meas. Sci. Technol. 20, 084019 (2009).

14. Zygo, "Compact optical wavelength compensator," http://www.zygo. com $/$ ? sup $=/$ resource/manuals.cgi?type $=z \mathrm{mi}$.

15. K. Meiners-Hagen and A. Abou-Zeid, "Refractive index determination in length measurement by two-colour interferometry," Meas. Sci. Technol. 19, 084004 (2008).

16. H. Schenke, "Optical methods for dimensional metrology in production engineering," Ann. CIRP 51, 685-699 (2007).

17. W. Estker, 'Large scale metrology: an update," Ann. CIRP 51, 587609 (2002)

18. D. Ellis, "Optical metrology techniques for dimensional stability measurements," Ph.D. dissertation (Technische Universiteit Delft, 2010).

19. E. Hecht and A. Zajac, Optics (Addison-Wesley, 1973), p. 2835.

20. J. Beers and T. Doiron, "Verification of revised water vapour correction to the index of refraction of air," Metrologia $2 \mathbf{2 9}, 315-316$ (1992).

21. Agilent (HP) "Operating manual of laser interferometer," http://cp. literature.agilent.com/litweb/pdf/05517-90108.pdf.

22. Zygo, "Operating manual of laser interferometer," http://www.zygo. $\mathrm{com} /$ ?/met/interferometers/.

23. Chapman MAV, "Environmental compensation of linear laser interferometer readings," Renishaw Technical white paper, TE329, 2013.

24. "Edlén or Ciddor? guidance in choosing the equation," http:// emtoolbox.nist.gov/Wavelength/Documentation. asp\#\#dlenorCiddor.

25. J. C. Tsai, "Ambient air refractive index measuring device," U.S. patent 5,394,244 (28 February 1995), https://www.google.com/patents/ US5394244.

26. J. Stone and A. Stejskal, "Using helium as a standard of refractive index: correcting errors in a gas refractometer," Metrologia 41, 189-197 (2004).

27. J. Stone and A. Stejskal, Wavelength tracking capabilities of a Fabry-Perot cavity," Proc. SPIE 5190, 327-337 (2003). 


\section{Chapter 9: Conclusion}

One advantage of the current developed refractometer is the simplicity of the design. From the discussions around the uncertainty contributions it is clear that the system has very few uncertainty contributors. Furthermore these do not have large contributions to the overall uncertainty. The system proves that a simple design with mainly commercial optics can be used to measure the refractive index to an accuracy of $1 * 10^{-8}$.

Two areas which can be investigated further are:

- From 6.2 the environmental range over which the system was tested, the air temperature and the pressure did not show any trends in the refractive index measurements. Further investigation of the effect of air temperature and air pressure, especially at the high altitude of NMISA, $1700 \mathrm{~m}$ above sea level can be performed. However for this to be done a special chamber will have to be conceptualised to control the air temperature and pressure.

- Lastly although this method can be used in everyday measurements to the required accuracy in place of using the weather station method, one major disadvantage is the use of the vacuum pump during the measurements where it generates heat which is not ideal in dimensional measurements. Further work will be to investigate a system which eliminates the use of a vacuum pump for the measurements. This would also simplify the method even further and allow for faster and slightly more accurate measurements. 


\section{Bibliography}

1. L, Kelvin. http://www.atmos.washington.edu/ robwood/teaching/451/Lord_Kelvin_quote.pdf.

2. Howart P, Redgarve F. Metrology in short. s.I. : ISBN 978-87-988154-5-7, 2008.

3. Role of Metrology in Conformity Assesment. BIPM presentation. 2015.

4. BIPM. Strategy 2013-2023 Consultative Committee for Length. 2013.

5. H, Schenke.Optical methods for dimensional metrology in production engineering. $685-699$, s.I. : Ann.CIRP, 2007, Vol. 51.

6. W, Estker.Large scale metrology: an update. 587-609, s.I. : Ann. CIRP, 2002, Vol. 51.

7. Company, Mckinsey \&. South Africa's Big Five: Bold priorities for Inclusive Growth. 2015.

8. J, Robinson.The Oxford Companion to Wine. 665-666, s.I. : Oxford University Press, 2006, Vol. 3.

9. Picard A, Fang H.Three methods to determing the density of moist air during mass comparisons. 31-40, s.l. : Metrologia, 2002, Vol. 39.

10. Picard A, Fang H.Methods to density moist air. s.I. : IEEE Transaction on Instrumentation and measurement, 2003.

11. F, Cardarelli. Encyclopaedia of Scientific Units, Weights and Measures. s.I. : Encyclopaedia of Scientific Units, Weights and Measures, 2003.

12. NPL. http://www.npl.co.uk/educate-explore/posters/history-of-length-measurement/. 2010.

13. Bruce C F, Hill R M.Wavenumder reproducibility of the radiation $2 p 10-5 d 5$ of Krypton 86. s.I. : Australian Journal of Physics, 1962, Vol. 15.

14. http://directedlight.com/laser-components/catalog/laser-lamps/. 2016.

15. Wilke T.Time to remeasure the metre. 258-263, s.I. : New Sceintist, 1983, Vol. 27.

16. Resolution 1. Metrologia. s.l. : Metrologia, 1984, Vol. 25.

17. Metrologia. metre, Practical realization of the definition of the. s.I. : Metrologia, 1997.

18. -. metre, Mise en Practique of the Definition of the. s.l. : Metrologia, 2006.

19. Edlén, B. The refractive index of air, Metrologia 2, 71-80 . 1965.

20. Downs, K. P. Birch and M. J.An updated Edlén equation for the refractive index of air. 155-162, s.I. : Metrologia, 1993, Vol. 30.

21. Ciddor, P. E.Refractive index of air: new equations for the visible and near infrared. 1566-1573, s.I. : Appl. Opt. , 1996, Vol. 35.

22. Bonsch G, Potulski E.Measurement of the refractive index of air and comparison with the modified Edlén's formulae. 35, s.l. : Metrologia, 1998, Vol. 35. 133-139.

23. Muijilwijk, R.Update of the Edlén formulae for the refractive index of air. 189, s.I. : Metrologia, 1988, Vol. 25.

24. Haitjema, H. Achieving traceability and sub-nanometer uncertainty using interferometric techniques. 084002, s.I. : Meas. Sci. Techno, 2008, Vol. 19.

25. E, Sommargren G. Apparatus for the measurement of the refractive index of a gas . US patent $4733967 \mathrm{~A}$. 1988.

26. $M$, Ishige.Measurement of air refractive index fluctuation from frequency change using a phase modulation homodyne interferometer and an external cavity laser diode. 084019, s.I. : Meas. Sci. Techno, 2009, Vol. 20.

27. Compact optical wavelength compensator. Zygo. s.l. :

http://www.zygo.com/?sup=/resource/manuals.cgi?type=zmi. .

28. Detro S M, Field A H. Wavelength tracking compensator for an interferometer . US Patent 4765741 A. 1988.

29. Lazar J,Suppression of air refractive index variations in high- resolution interferometry. 7644-7655, s.I. : Sensors, 2011, Vol. 11.

30. Lazar J, Refractive index compensation in over-determined interferometric systems. $14084340-14094$, s.l. : Sensors, 2012, Vol. 12.

31. Meiners-Hagen $\mathrm{K}$, Abou-Zeid ,Refractive index determination in length measurement by two-colour

$$
\text { Page | } 51
$$


interferometry. A. 084004, s.I. : Meas. Sci. Techno, 2008, Vol. 19.

32. Ellis D, Optical mtrology techniques for dimensional stability measurements. s.I. : Ph.D. dissertation ,Technische Universiteit Delf, 2010, Vol. 356.

33. Lewis, A. Absolute length measurement using multiple wavelength phase setting interferometry. PhD Thesis, 1993.

34. Hecht E, Zajac A. Optics, Vol. 36. s.I. : Addison-Wesley, 2002.

35. Beers J, Doiron T., "Verification of revised water vapour correction to the index of refraction of air. 315 316 , s.l. : Metrologia, 1992, Vol. 29.

36. Operating manual of laser interferometer. (HP), Agilent. s.I. :

http://cp.literature.agilent.com/litweb/pdf/05517-90101.pdf.

37. -. Zygo. s.l. : http://www.zygo.com/?/met/interferometers.

38. MAV, Chapman, Environmental compensation of linear laser interferometer readings.. Renishaw Technical white paper, 2013, Vol. TE329.

39. 1, Geometrical product specifications (GPS) ISO. Standard reference temperature for the specification of geometrical and dimensional properties. s.I. : ISO, 2016.

40. J, Stone. Edlén or Ciddor? guidance in choosing the equation.

http://emtoolbox.nist.gov/Wavelength/Documentation.asp\#EdlenorCiddor.

41. M, Chapman. Heterodyne and homodyne interferometry. http://www.renishaw.com/en/interferometryexplained. 7854 .

42. Terrien JAn air refractometer for interference length metrology. 3, s.I. : Metrologia, 1965, Vol. 1.

43. Tsai J C. Ambient air refractive index measuring device. U.S. patent 5,394,244.

https://www.google.com/patents/US5394244, 1995.

44. Schellekens P, Wilkening G, Reinboth F, Downs M J, Brirch K P, Spronck J.Measurements of the refractive index of air using interference refractometers. 249-287, s.I. : Metrologia, 1986, Vol. 22.

45. properties, Glass. http://glassproperties.com/expansion/ExpansionMeasurement.htm.

46. Stone J, Stejskal A. Using helium as a standard of refractive index: correcting errors in a gas refractometer. $189374-197$, s.l. : Metrologis, 2004, Vol. 41.

47. Stone J, Stejskal A Wavelength tracking capabilities of a Fabry-Perot cavit.. 5190, 327, s.I. : Proc. SPIE , 2003.

48. The International System of Units (SI). Units, The International System of. 8th Edition, 2003, Vol. 35. 49. Chetty N, Kruger OA. Robust air refractometer for accurate compensation of the refractive index of air in everyday use. 9118-9122, s.I. : OSA, 2016, Vol. 55.

50. JCGM, WG 1. Evaluation of measurement data. 1, 2008.

51. Hidnert PThermal Expansion of Five Selected Optical Glasses. 311-312, s.l. : Journal of Research of the National Bureau of Standards, 1954, Vol. 52.

52. Linear-expansion-coefficients. http://www.engineeringtoolbox.com/linear-expansion-coefficientsd_95.html.

53. Giacomo P,Equation for the Detemination of the Deinsity of moist air. 33-40, s.l. Metrologia, 1981, Vol. 18.

54. Davis S R, Eguation for the Detertimination od density of moist air. 67-70, 1992, Vol. 29. 


\section{Appendix I}

Calculation of Refractive index, step by step method, according to Bonsch (18):

To enable a quick calculation of the refractive index of air on the basis of the fitted experimental values without

a detailed study of the whole paper, the complete set of equations is repeated and concise instructions are

outlined here.

The calculation starts with the dispersion formula of dry air for the new standard conditions, temperature

C (ITS-90), pressure $100000 \mathrm{~Pa}$ and $0.04 \%$ CO2 content, describing the refractivity of standard air dependent on the wavenumber $\sigma=1 / \lambda$, where $\lambda$ ?designates wavelength:

$$
(n-1)_{N} * 10^{-8}=8091,37+2333983-130-\left(\sigma / \mu m-1+15518 /\left\{38,9-\left(\frac{\sigma}{\mu m-1}\right) 10^{-6} .\right.\right.
$$

A CO2 content $x$, differing from $0,04 \%$, changes the refractivity to

$$
\left.(n-1)_{x}=(n-1)_{N *}\{1+0,5327 * x-0,0004)\right\}
$$

The deviation of temperature and pressure from the reference conditions is taken into account by

$$
(n-1)_{T p}=\frac{p(n-1)_{s}}{93214,60} * \frac{1+10^{-8} p(0,5953-0,009876 t)}{\left(1+0,0036610_{t}\right)}
$$

In the last step, the influence of water vapour with partial pressure $f$ is calculated, which results in the refractive

index $\eta_{t p f}$ for moist air under total pressure $p$ and for temperature $t$ and CO2 content $x$ :

$$
\eta_{t p f}-\eta_{t p}=-f *\left(3,7345-0,0401 \lambda^{2}\right) * 10^{-10} .
$$

For the transformation of dew-point temperatures to water-vapour pressure, the equation for the saturation pressure of water $p_{s v}$ given by (49) with the updated constants of (13) has been used:

$$
p_{s v}=1 * \exp \left(\mathrm{AT}^{2}+\mathrm{BT}+\mathrm{C}+\mathrm{D}\right)
$$

with

$\mathrm{A}=123788747 * 10^{-5}, \mathrm{~B}=-1,9121216 * 10^{-2}, \mathrm{C}=33,93711047, \mathrm{D}-6,3431645 * 10^{-3}$ 


\section{Appendix II}

Edlén Calculation of the Index of Refraction as describe by J Stone (40):

(a) Preliminaries:

- Convert all temperatures to Celsius.

- Convert all pressures to Pascal.

- Calculate partial pressure $p_{\mathrm{v}}$ as described previously.

(b) Define constants:

$A=8342.54 \quad B=2406147$

$C=15998 \quad D=96095.43$

$E=0.601 \quad F=0.00972 \quad G=0.003661$

(c) Convert the laser vacuum wavelength $\lambda$ to micrometers and then find

$S=1 / \lambda^{2}$

(d) Calculate intermediate results at air pressure $p$, water vapor partial pressure $p_{\mathrm{v}}$, and temperature $t$ :

$n_{s}=1+10^{-8}[A+B /(130-S)+C /(38.9-S)]$

$X=\left[1+10^{-8}(E-F t) p\right] /(1+G t)$

$n_{t y}=1+p\left(n_{s}-1\right) X / D$

(e) Calculate the final result for index of refraction $n$, using our variation of the water vapour term:

$n=n_{t y}-10^{-10}[(292.75) /(t+273.15)] \times[3.7345-0.0401 S] \times p_{v}$ 